\title{
INVENTORY MODELS WITH INTEGRATED TIME DEPENDENT DEMANDS FOR DETERIORATING ITEMS - IN THIRD AND FOURTH ORDER EQUATIONS
}

\author{
C.K. Sivashankari And Lalitha RamachandRan*
}

\begin{abstract}
Inventory models with integrated time-dependent demands for deteriorative items are considered in this study. The demand models found in the literature include constant, linear, quadratic, exponential, price dependent, and stock dependent among others. To wit, no study exists that uses integrated time-dependent demands. Three models are developed: The first model uses continuously compounded demands, the second model uses linear demands integrated with continuously compounded demands, and the third model uses quadratic demands integrated with continuously compounded demands. Mathematical models are delineated for each model and relevant examples are provided to elucidate the proposed procedure. The objective herein is to obtain optimum order quantities and order intervals concerning the overall cost. Sensitivity analysis is provided for each of the three models. The necessary data was generated using Visual Basic 6.0.
\end{abstract}

Mathematics Subject Classification. 90B05.

Received March 7, 2021. Accepted June 30, 2021.

\section{INTRODUCTION}

Inventory models are of primary significance in industries that require manufacturing, distribution, and retail infrastructure. Among the many concerns, demand plays a critical role in determining the best inventory strategy. Classical inventory models, from a boundless forecasting perspective, presume constant demand but this assumption is only effective for a determinate period during the mature phase of the product life-cycle. In other phases, the demand for the product may be growing, for example, after the product is launched into the market, or declining, perhaps due to new competition. Addressing changing demand involves research in two aspects of inventory planning models; the deterioration of inventory items, and variation in the demand rate over time. It is, obvious that demand is not constant; it may be time-dependent. Research has developed inventory models that presume constant, linearly increasing or decreasing and, quadratically increasing or decreasing demand for items; they also consider, ramp type demand, and stock dependent models, among others. A significant number of authors have performed extensive work on real-life inventory control issues based on exponential and linear demand. Aggarwal and Jaggi [1] considered an inventory model with an exponential deterioration rate

Keywords. Integrated demands, linear with exponential, quadratic with exponential, optimality, sensitivity analysis, higher order equations.

Department of Mathematics, R.M.K. Engineering College, Kavaraipetti 601206, India.

*Corresponding author: lar.sh@rmkec.ac.in 
under permissible payment delay conditions. Shukla et al. [18] developed an inventory model for deteriorating products with exponential time-dependent demand rates, shortages allowed, and partial backlogging. Taylor's used series expansion to find a closed-form optimal solution. Khanna et al. [7] developed an inventory model for deteriorating items with time-dependent demand where payment delays are acceptable. While the deterioration rate is presumed to be constant, the time-varying demand rate is considered to be a quadratic function of time. Goswami and Chaudhuri [6] developed an economic order quantity (EOQ) inventory model for an item subject to deterministic time-dependent demand with a linear (positive) trend. Presuming a uniform rate of inventory replenishment, they solved the model, allowing for no shortages and inventory shortages. Trailogyanath Singh et al. [21] developed an inventory model to determine the EOQ for a deteriorating item with a linear time function for the demand rate, a time-proportional deterioration rate, and shortages not allowed. They implement the concept of integrated demands in a higher-order equation for forms of demands such as linear or quadratic continuously compounded demand.

Realizing that linear, quadratic, exponential, ramp type, stock-dependent, and price-dependent demand, along with other patterns, do not precisely depict demand for certain products, this paper adopts the concept of integrating time-dependent demand in a higher-order equation such as linear with continuously compounded demand $(a+b t) e^{R t}$ and quadratic with continuous compounded demand $\left(a+b t+c t^{2}\right) e^{R t}$. As far as previous research is concerned, no author has researched integrated time-dependent demands. In view of this feature, three models are developed here: the first uses continuously compounded demand, the second uses linear demand integrated with continuously compounded demand, and the third uses quadratic demands integrated with continuously compounded demand. For the three models, the mathematical derivation is generated, numerical examples demonstrated, and data provided using Visual Basic 6.0. This paper aims to present inventory models with integrated time-dependent demands for deteriorating items in a higher-order equation; its objective is thus to develop mathematical models to determine the optimal quantity and optimal timing of inventory replenishment to meet future demand based on integrated time-dependent demands.

The paper is further organized as follows: The relevant literature is reviewed in Section 2, and assumptions and notations for the development of the model are provided in Section 3. The inventory model is formulated and the optimal solution process is developed in Section 4. A comparison of continuously compounded demand, linear demand integrated with continuously compounded demand, and quadratic demand integrated with continuously compounded demand is provided in Section 5 and finally, the summary and the scope for future research are given in Section 6.

\section{Literature REVIEW}

Baker and Urban [3] developed deterministic and continuous inventory models in which the demand rate depends upon the inventory, and the demand rate for the item has a polynomial functional form. Mandal and Phaujdar [9] determined a uniform rate of production and stock-dependent demand for deteriorating items, where shortages are permitted, and excess demand creates a backlog. Pal et al. [11] determined a stock-dependent inventory model with a constant deterioration rate, determining the average net profit $\pi$ over one production run, and optimizing the decision variables $Q$ (initial stock) and $T$ (duration of a production cycle). In 1995, Chung et al. [4] proposed inventory models for deteriorating items with stock-dependent sales rates and derived profit functions without backlogging and with complete backlogging. They explore the efficient use of the Newton-Raphson method when finding optimum solutions for-profit functions per unit time modeled in both contexts. Srivastava and Gupta [23] developed an infinite time-horizon inventory model for deteriorating items, assuming that the demand rate is constant for some time and then a linear function of time. They obtain the theoretical expressions for the optimal inventory level and total average cost, as a function of the sales price and time-dependent holding cost. Ajanta Roy [13] developed an inventory model with a time-proportional rate of deterioration and demand. Vinod Kumar et al. [10] developed an inventory model with time-dependent demand, and a time-varying holding cost, and time-proportional deterioration. The model allowed for inventory shortages with partial backlogging. An EOQ inventory model was developed by Shukla et al. [19] with quadratic demand 
that permitted both payment delay and shortages. Tripathi and Manjit Kaur [24] considered an inventory model with deteriorating items to be a phenomenon that cannot be overlooked, as failing to consider such context may provide an absurd result. In the high-tech business industry, deterioration is not necessarily constant, but rather time-dependent. Trailokyanath Singh et al. [20] introduced a model of the EOQ for deteriorating items with a deterioration rate proportional to time, a time-demand ramp-type demand rate, and shortages. The model allowed completely backlogged shortages and the ramp-type demand rate is deterministic, changing over time to a certain point and then constant. Saha and Sen [14] proposed an inventory model with a sale price and time-dependent demand, a constant holding cost, and time-dependent deterioration. Shaikh et al. [16] considered purchase cost irrespective of the order size and carrying cost over the entire cycle period, treating deterioration as another imperative issue in inventory analysis, because of its huge impact on the profit or cost of the inventory system. Considering these factors, they developed two different inventory models: (a) an inventory model for a zero ending case and (b) an inventory model for a shortage case. Tripathi et al. [25] established an inventory model of exponential time-dependent demand and time-dependent deterioration. The model accounts for shortages and considers a proportionate demand rate and unit production cost. Khedlekar et al. [8] attempted to develop a method of optimization an economic production quantity model for deteriorating items with production disruption. They determined the optimum production time before and after the system disruption. They have also developed a model that optimizes product shortages, which is useful for determining the time to start and finish production when the system gets disrupted. Shaikh et al. [17] developed an inventory model based on the sale price of deteriorating items with variable-demand allowing for shortages and the advertising of items under financial trade credit policy. Sivasankari [22] proposed an inventory model for deteriorating items with under-inflation time-dependent exponential demand and developed an optimal solution from higherorder equations. Pervin et al. [12] formulated an inventory model for deteriorating items. To minimize the rate of deterioration, they apply preservation technologies and quantify the level of expenditure on preservation technology. In their model, the demand function depends on the stock level and price, and the production rate is linearly time-dependent, based on consumer demand with shortages permitted. Sarkret et al. [15] investigated an integrated vendor-buyer model with shortages under a stochastic lead time, which is assumed to be variable but depends on the buyer's order size and the vendor's production rate; the proposed model determines the net present value of the expected total cost. Ahmad and Benkherout [2] proposed a procedure for determining the optimal replenishment policy for a basic inventory model of stock-dependent demand items, non-instantaneous deteriorating items, and partial backlogging. Dong et al. [5] proposed a problem that focuses on determining the optimal price of the existing product and the inventory level for the new product. Inspired by practice, the problem considers various strategies for the existing product and the cross-elasticity of demand for existing and new products.

\section{Assumptions And notations}

\subsection{Assumptions}

(1) The initial inventory level is zero. (2) The demand rate is continuous compound demand $Y=Y e^{R t}$ in model 1, the demand rate is integrated linear demand with continuous compound demand that is, $Y=(a+b t) e^{R t}$ in model 2 and the demand rate is integrated quadratic demand with continuous compound demand that is, $Y=\left(a+b t+c t^{2}\right) e^{R t}$ in model 3, where $R>0$ rate of demand and it is a continuous function of time, "a" be the initial demand and "b" and "c" are demand based on time. (3) The deteriorating rate is constant. (4) The planning horizon is finite. (5) Lead time is zero. (6) There is no repair or replacement of the deteriorated items. (7) Shortages are not considered in this model.

\subsection{Notations}

(1) $Y=Y e^{R t}$ - demand is continuous compound demand rate in units per unit time, $Y=(a+b t) e^{R t}$ - linear demand integrated with continuous compound demand in units in unit time and $Y=\left(a+b t+c t^{2}\right) e^{R t}$ - quadratic 


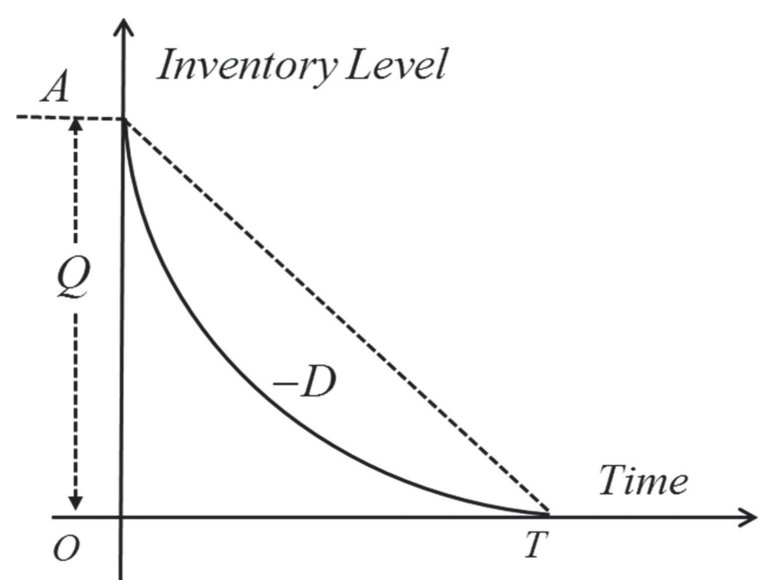

FiguRE 1. Economic lot size model for deteriorating items with continuous compound demand.

demand integrated with continuous compound demand in units in unit time, (2) $Q^{*}$ - optimal inventory size, (3) $\mu$ - the rate of deteriorative. (4) $D_{C}$ - deterioration cost per unit, (5) $S_{C}$ - ordering cost/order, (6) $r$ - the rate of interest, (6) $H_{C}$ - holding cost per unit/time, (7) $T_{1}$ - the time during which the inventory is building up, (8) $T$ - cycle time, (9) TC - total cost, (10) $R$ - rate of increase in demand.

\section{Mathematical models}

\subsection{EOQ inventory model for deteriorative items with continuous compound demand}

This model is developed for deteriorating inventory model in which demand is continuous compound demand with a function of time that is $Y=Y e^{R t}$, where $R>0$ at time $t$ and "R" stands for the increase in demand. Let $Q$ be the units of item arrive at the inventory system at the beginning of each cycle. The inventory level decreases due to demand and deteriorating till it becomes zero in the interval $(0, T)$. The total process is repeated. The inventory level at different instants of time is shown in Figure 1. The differential equation describing the instantaneous states of $I(t)$ in the interval $[0, T]$ is given below:

$$
\frac{\mathrm{d}}{\mathrm{d} t} I(t)+\mu I(t)=-Y e^{R t} ; \quad 0 \leq t \leq T
$$

The basic boundary conditions in the differential equation,

$$
I(0)=Q \quad \text { and } \quad I(T)=0 .
$$

From the equation (4.1),

$$
I(t)=\frac{Y}{R+\mu}\left(e^{(R+\mu) t-\mu t}-e^{R t}\right) .
$$

Total cost $\mathbf{T C}(\boldsymbol{T})$ : total cost comprised of the sum of the setup cost, holding cost, and deteriorating cost. They are grouped after evaluating the above cost individually.

$$
\begin{aligned}
\text { Setup cost } & =\frac{S_{C}}{T} . \\
\text { Holding cost } & =\frac{H_{C}}{T} \int_{0}^{T} \frac{Y}{R+\mu}\left(e^{(R+\mu) T-\mu t}-e^{R t}\right) \mathrm{d} t
\end{aligned}
$$




$$
\begin{aligned}
& =\frac{-Y H_{C}}{R \mu(R+\mu) T}\left(\operatorname{Re}^{R T}+\mu e^{R T}-\operatorname{Re}^{(R+\mu) T}-\mu\right) \\
& =\frac{-Y H_{C}}{R \mu(R+\mu) T}\left(R\left(e^{R T}-e^{(R+\mu) T}\right)+\mu\left(e^{R T}-1\right)\right) \\
& =\frac{Y H_{C}}{R \mu(R+\mu) T}\left(R\left(e^{(R+\mu) T}-e^{R T}\right)+\mu\left(1-e^{R T}\right)\right) .
\end{aligned}
$$

(3) Deteriorating cost $=\frac{Y \mu D_{C}}{R \mu(R+\mu) T}\left(R\left(e^{(R+\mu) T}-e^{R T}\right)+\mu\left(1-e^{R T}\right)\right)$

Total cost, $\operatorname{TC}(T)=\frac{H_{C}}{T}+\frac{Y\left(H_{C}+\mu D_{C}\right)}{R \mu(R+\mu) T}\left[R\left(e^{(R+\mu) T}-e^{R T}\right)+\mu\left(1-e^{R T}\right)\right]$.

\section{Optimality conditions:}

$$
\frac{\mathrm{d}}{\mathrm{d} T} \mathrm{TC}(T)=0 \quad \text { and } \quad \frac{\mathrm{d}^{2}}{\mathrm{~d} T^{2}} \mathrm{TC}(T)>0 .
$$

Differentiate the equation (4.7) with respect to $T$

$$
\begin{aligned}
\frac{\mathrm{d}}{\mathrm{d} T} \mathrm{TC}(T)=-S_{C}+\frac{Y\left(H_{C}+\mu D_{C}\right)}{R \mu(R+\mu)}\left[\begin{array}{l}
T\left\{R(R+\mu) e^{(R+\mu) T}-\operatorname{Re}^{R T}\right\}-R\left(e^{(R+\mu) T}-e^{R T}\right) \\
+T\left\{\mu\left(-\operatorname{Re}^{R T}\right)\right\}-\mu\left(1-e^{R T}\right)
\end{array}\right] & =0 \\
R(R+\mu) T e^{(R+\mu) T}-R T e^{R T}-R\left(e^{(R+\mu) T}-e^{R T}\right)-R \mu T e^{R T}-\mu\left(1-e^{R T}\right) & =\frac{S_{C} R \mu(R+\mu)}{Y\left(H_{C}+\mu D_{C}\right)} .
\end{aligned}
$$

This is an optimum solution of $T$ in a higher-order equation. This equation can be evaluated by using Matlab. But the reader's convenient; the equation is reduced to the fourth-order equation then third order equation in $T$.

On simplification

$$
\left[\begin{array}{l}
\frac{\mu T^{2}}{2}+\frac{R T^{2}}{2}+\frac{2 R^{2} T^{3}}{3}+R \mu T^{3}+\frac{\mu^{2} T^{3}}{3} \\
+\frac{3 R^{3} T^{4}}{8}+\frac{3 R^{2} \mu T^{4}}{4}+\frac{R \mu^{2} T^{4}}{2}+\frac{\mu^{3} T^{4}}{8}
\end{array}\right]=\frac{S_{C}(R+\mu)}{Y\left(H_{C}+\mu D_{C}\right)}
$$

On simplifications,

$$
\begin{aligned}
\frac{1}{2}(R+\mu) T^{2}+\left(\frac{2 R^{2}}{3}+R \mu+\frac{\mu^{2}}{3}\right) T^{3}+\left(\frac{3 R^{3}}{8}+\frac{3 R^{2} \mu}{4}+\frac{R \mu^{2}}{2}+\frac{\mu^{3}}{8}\right) T^{4} & =\frac{S_{C}(R+\mu)}{Y\left(H_{C}+\mu D_{C}\right)} \\
{\left[\begin{array}{l}
3\left(3 R^{3}+6 R^{2} \mu+4 R \mu^{2}+\mu^{3}\right) T^{4} \\
+8\left(2 R^{2}+3 R \mu+\mu^{2}\right) T^{3}+12(R+\mu) T^{2}
\end{array}\right] } & =\frac{24(R+\mu) S_{C}}{Y\left(H_{C}+\mu D_{C}\right)}
\end{aligned}
$$

which is optimal solution for cycle time $T$ in forth order equation and it is reduced to 3th order equation.

$$
2\left(2 R^{2}+3 R \mu+\mu^{2}\right) T^{3}+3(R+\mu) T^{2}=\frac{6(R+\mu) S_{C}}{Y\left(H_{C}+\mu D_{C}\right)} .
$$

Note: when higher power values are assigned zero, then the equation (4.4) reduces to basic inventory model $T=\sqrt{\frac{2 S_{C}}{Y\left(H_{C}+\mu D_{C}\right)}}$.

(Appendix A)

Numerical example: let us consider the cost parameters

$$
Y=5000 \text { units, } H_{C}=7, D_{C}=50, S_{C}=150, \mu=0.01, R=0.1 .
$$


TABLE 1. Variation of rate of deteriorating items with inventory and total cost and continuous compound demand.

\begin{tabular}{lllllll}
\hline \hline$\mu$ & $T$ & $Q$ & Setup cost & Holding cost & Deteriorating cost & Total cost \\
\hline 0.01 & 0.0888 & 444.45 & 1687.45 & 1565.27 & 111.80 & 3364.53 \\
0.02 & 0.0860 & 430.30 & 1742.94 & 1515.57 & 216.51 & 3475.03 \\
0.03 & 0.0834 & 417.42 & 1796.74 & 1470.32 & 315.06 & 3582.14 \\
0.04 & 0.0811 & 405.62 & 1849.00 & 1428.83 & 408.25 & 3686.15 \\
0.05 & 0.0789 & 394.77 & 1899.83 & 1390.75 & 496.71 & 3787.33 \\
0.06 & 0.0769 & 384.74 & 1949.36 & 1355.57 & 580.96 & 3885.89 \\
0.07 & 0.0750 & 375.43 & 1997.67 & 1322.90 & 661.45 & 4075.92 \\
0.08 & 0.0733 & 366.77 & 2044.86 & 1292.49 & 738.50 & 4167.71 \\
0.09 & 0.0717 & 358.67 & 2091.00 & 1264.08 & 812.62 & 4257.53 \\
0.10 & 0.0702 & 351.09 & 2136.16 & 1237.46 & 883.90 & \\
\hline
\end{tabular}

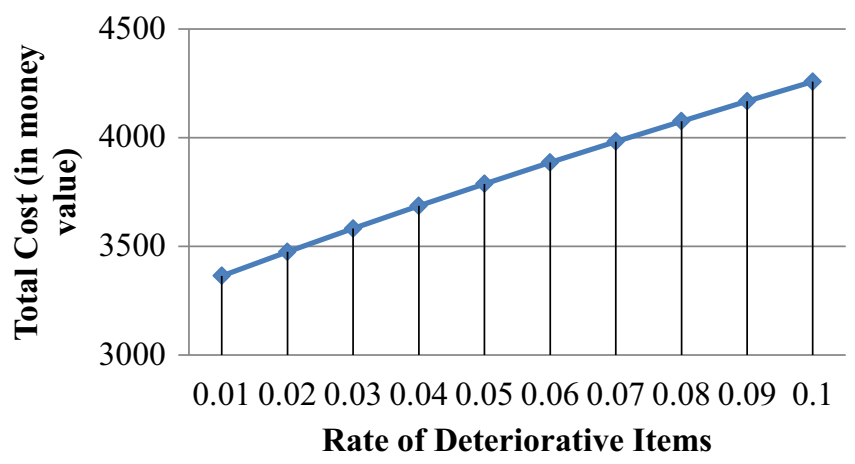

FigURE 2. Relationship between rate of deteriorative items with total cost in continuous compound demand.

\section{Optimum solution}

The cubic equation is $0.0462 T^{3}+0.33 T^{2}-0.00264=0$ in which one positive real root and two negative real roots. That is, $T=0.08889,-7.14174$ and -0.09001 . The positive root $T=0.0888$ is considered in this model. Therefore, Cycle time $=0.0888$, Optimum Quantity $Q^{*}=444.45$, Setup cost $=1687.45$, Holding cost $=$ 1565.27, Deteriorating cost $=111.80$, Total cost $=3364.53$.

In above Table 1, it is observed that a study of the rate of the deteriorative items with cycle time, optimum quantity, setup cost, deteriorating cost, and total cost. There is a positive relationship between the increase in the rate of deterioration for items $\mu$ and setup costs, deteriorating costs, and total costs, while there is a negative relationship between the increase in the rate of deterioration for items $\mu$ and cycle time, optimum quantity, and holding cost.

The graphical representations between total cost with rate of deteriorative items is given in Figure 2:

\section{Sensitivity analysis}

The total cost functions are the real solution in which the model parameters are assumed to be static values. It is reasonable to study the sensitivity i.e. the effect of making changes in the model parameters over a given optimum solution. It is important to find the effects on different system performance measures, such as cost function, inventory system, etc. For this purpose, sensitivity analysis of various system parameters for the models 
TABLE 2. Effect of Demand and cost parameters on optimal values in continuous compound demand.

\begin{tabular}{|c|c|c|c|c|c|c|c|}
\hline \multirow{2}{*}{\multicolumn{2}{|c|}{ Cost parameters }} & \multicolumn{6}{|c|}{ Optimal values } \\
\hline & & $T$ & $Q$ & Setup cost & Holding cost & Deteriorative cost & Total cost \\
\hline \multirow{5}{*}{$S_{C}$} & 130 & 0.0827 & 413.94 & 1570.27 & 1457.19 & 104.09 & 3131.54 \\
\hline & 140 & 0.0858 & 429.47 & 1629.89 & 1512.19 & 108.01 & 3250.11 \\
\hline & 150 & 0.0888 & 444.45 & 1687.45 & 1565.27 & 111.80 & 3364.53 \\
\hline & 160 & 0.0917 & 458.94 & 1743.14 & 1616.61 & 115.47 & 3475.23 \\
\hline & 170 & 0.0945 & 472.92 & 1797.14 & 1666.36 & 119.02 & 3582.53 \\
\hline \multirow{5}{*}{$H_{C}$} & 5 & 0.1036 & 518.48 & 1446.52 & 1305.61 & 130.56 & 2882.70 \\
\hline & 6 & 0.0954 & 477.20 & 1571.64 & 1441.18 & 120.09 & 3132.92 \\
\hline & 7 & 0.0888 & 444.45 & 1687.45 & 1565.27 & 111.80 & 3364.53 \\
\hline & 8 & 0.0835 & 417.64 & 1795.76 & 1680.36 & 105.02 & 3581.15 \\
\hline & 9 & 0.0790 & 395.17 & 1897.87 & 1788.14 & 99.34 & 3785.36 \\
\hline \multirow{5}{*}{$D_{C}$} & 30 & 0.0901 & 450.46 & 1664.94 & 1586.57 & 67.99 & 3319.51 \\
\hline & 40 & 0.0895 & 447.43 & 1676.23 & 1575.81 & 90.46 & 3342.10 \\
\hline & 50 & 0.0888 & 444.45 & 1687.45 & 1565.27 & 111.80 & 3364.53 \\
\hline & 60 & 0.0883 & 441.54 & 1698.89 & 1554.94 & 133.28 & 3386.82 \\
\hline & 70 & 0.0877 & 438.68 & 1709.66 & 1544.81 & 154.48 & 3408.96 \\
\hline \multirow{10}{*}{$R$} & 0.05 & 0.0891 & 445.75 & 1682.52 & 1565.25 & 111.80 & 3359.58 \\
\hline & 0.10 & 0.0888 & 444.45 & 1687.45 & 1565.27 & 111.80 & 3364.53 \\
\hline & 0.15 & 0.0886 & 443.17 & 1692.34 & 1565.31 & 111.80 & 3369.46 \\
\hline & 0.20 & 0.0883 & 441.90 & 1697.18 & 1565.35 & 111.81 & 3374.35 \\
\hline & 0.25 & 0.0881 & 440.66 & 1701.99 & 1565.41 & 111.81 & 3379.22 \\
\hline & 0.30 & 0.0878 & 439.42 & 1706.75 & 1565.48 & 111.82 & 3384.06 \\
\hline & 0.35 & 0.0876 & 438.21 & 1711.48 & 1565.57 & 111.82 & 3388.88 \\
\hline & 0.40 & 0.0874 & 437.01 & 1716.17 & 1565.66 & 111.83 & 3393.66 \\
\hline & 0.45 & 0.0871 & 435.83 & 1720.82 & 1565.76 & 111.84 & 3398.43 \\
\hline & 0.50 & 0.0869 & 434.67 & 1725.43 & 1565.88 & 111.84 & 3403.16 \\
\hline
\end{tabular}

of this research is required to observe whether the current solutions remain unchanged, the current solutions become infeasible, etc.

Managerial insights: a sensitivity analysis is performed to study the effects of changes in the system parameters, rate of deteriorating items $\mu$, ordering cost per order $\left(S_{C}\right)$, holding cost per unit time $\left(H_{C}\right)$, deteriorating cost per unit time $\left(D_{C}\right)$, and rate of increase in demand $(R)$ on optimal values that is optimal cycle time $(T)$, optimal quantity $(Q)$, setup cost, holding cost, deteriorating cost and total cost. The sensitivity analysis is performed by changing (increasing or decreasing) the parameter taken at a time, keeping the remaining parameters at their original values. The following influences can be obtained from sensitivity analysis based on Table 2 .

(1) There is a positive relationship between the increase in the setup costs per set $\left(S_{C}\right)$ and the cycle time, the optimum quantity, setup costs, holding costs, deteriorating costs, and total costs.

(2) There is a positive relationship between the increase in the holding cost per unit time $\left(H_{C}\right)$ and the setup cost, holding cost, and total cost while there is a negative relationship between the increase in the holding cost per unit time $\left(H_{C}\right)$ and the cycle time, optimal quantity and deteriorating cost.

(3) There is a positive relationship between the increase in the rate of demand $(R)$ and the setup cost, holding cost, deteriorative cost and total cost, while there is a negative relationship between the increase in the rate of demand $(R)$ and the cycle time, and optimum quantity.

(4) Similarly, other parameters, rate of deteriorating items $\left(D_{C}\right)$ can also be observed from Table 2. 


\subsection{EOQ inventory model for deteriorating items integrated with linear and continuous compound demand}

This model is developed for deteriorating inventory model in which demand is linear integrated with continuous compound demand which is a function of time that is $Y=(a+b t) e^{R t}$, where $R>0$ at time $t$ and "R" stands for the increase in demand, "a" stands for initial demand, and "b" demand based on time. Let $Q$ be the units of the item arriving at the inventory system at the beginning of each cycle. The inventory level decreases due to demand and deteriorating till it becomes zero in the interval $(0, T)$. The total process is repeated. The differential equation describing the instantaneous states of $I(t)$ in the interval $[0, T]$ is given below:

$$
\begin{aligned}
\frac{\mathrm{d}}{\mathrm{d} t} I(t)+\mu I(t) & =-(a+b t) e^{R t} ; \quad 0<t<T_{1} \\
I(o) & =Q, I(T)=0 .
\end{aligned}
$$

From the differential equation (4.11)

$$
I(t)=\frac{1}{R+\mu}\left[(a+b T) e^{(R+\mu) T-\mu t}-(a+b t) e^{R t}\right]+\frac{b}{(R+\mu)^{2}}\left[e^{R t}-e^{(R+\mu) T-\mu t}\right] .
$$

\section{Note:}

(i) When $a=Y$ and $b=0$, then $I(t)=\frac{Y}{R+\mu}\left[e^{(R+\mu) T-\mu t}-e^{R t}\right]$ which is continuous compound demand. (as per model 1)

(ii) When $R=0$, then $I(t)=\left(\frac{a}{\mu}+\frac{b T}{\mu}-\frac{b}{\mu^{2}}\right) e^{(T-t) \mu}-\frac{a}{\mu}-\frac{b t}{\mu}+\frac{b}{\mu^{2}}$ which is Linear Demand. (Appendix B)

(iii) When $a=Y$ and $b=0$, then $\left.I(t)=\frac{Y}{\mu}\left(e^{(T-t) \mu}-1\right)\right)$ constant Demand.

(Appendix A)

To find $Q$ :

$$
I(0)=Q \Rightarrow Q=\frac{1}{R+\mu}\left[(a+b T) e^{(R+\mu) T}-a\right]+\frac{b}{(R+\mu)^{2}}\left[1-e^{(R+\mu) T}\right] .
$$

On simplification,

$$
Q=a T+\frac{1}{2} a(R+\mu) T^{2}+\frac{b T^{2}}{2} .
$$

Total cost $\mathbf{T C}(\boldsymbol{T})$ : total cost comprised of the sum of the setup cost, holding cost, and deteriorating cost. They are grouped after evaluating the above cost individually.

Total cost $(\mathrm{TC})=$ Setup cost + holding cost + deteriorating cost.

$$
\begin{aligned}
\text { Setup cost }= & \frac{S_{C}}{T} . \\
\text { (2) } \quad \text { Holding cost }= & \frac{H_{C}}{T}\left[\int_{0}^{T} \frac{1}{R+\mu}\left[(a+b T) e^{(R+\mu) T-\mu t}-(a+b t) e^{R t}\right]\right. \\
& \left.+\frac{b}{(R+\mu)^{2}}\left[e^{R t}-e^{(R+\mu) T-\mu t}\right]\right] \mathrm{d} t \\
= & \frac{H_{C}}{T}\left[\begin{array}{l}
\frac{1}{R^{2} \mu(R+\mu)}\left[\begin{array}{l}
R^{2}(a+b T)\left(e^{(R+\mu) T}-e^{R T}\right)+b \mu\left(e^{R T}-1\right) \\
+R a \mu\left(1-e^{R T}\right)-R b T e^{R T}
\end{array}\right] \\
+\frac{b}{R \mu(R+\mu)^{2}}\left[\mu\left(e^{R T}-1\right)+R\left(e^{R T}-e^{(R+\mu) T}\right)\right] .
\end{array}\right] \\
\text { (3) Deteriorative cost }= & \frac{\mu D_{C}}{T}\left[\begin{array}{l}
\frac{1}{R^{2} \mu(R+\mu)}\left[\begin{array}{l}
R^{2}(a+b T)\left(e^{(R+\mu) T}-e^{R T}\right)+b \mu\left(e^{R T}-1\right) \\
+R a \mu\left(1-e^{R T}\right)-R b T e^{R T}
\end{array}\right] \\
+\frac{b}{R \mu(R+\mu)^{2}}\left[\mu\left(e^{R T}-1\right)+R\left(e^{R T}-e^{(R+\mu) T}\right)\right]
\end{array}\right] .
\end{aligned}
$$


Therefore, total cost,

$$
\mathrm{TC}=\frac{S_{C}}{T}+\frac{H_{C}+\mu D_{C}}{T}\left[\begin{array}{l}
\frac{1}{R^{2} \mu(R+\mu)}\left[\begin{array}{l}
R^{2}(a+b T)\left(e^{(R+\mu) T}-e^{R T}\right)+b \mu\left(e^{R T}-1\right) \\
+R a \mu\left(1-e^{R T}\right)-R b \mu T e^{R T}
\end{array}\right] \\
+\frac{b}{R \mu(R+\mu)^{2}}\left[\mu\left(e^{R T}-1\right)+R\left(e^{R T}-e^{(R+\mu) T}\right)\right]
\end{array}\right] .
$$

\section{Optimality conditions:}

$$
\frac{\mathrm{d}}{\mathrm{d} T} \mathrm{TC}(T)=0 \quad \text { and } \quad \frac{\mathrm{d}^{2}}{\mathrm{~d} T^{2}} \mathrm{TC}(T)>0
$$

Differentiate the equation (4.16) with respect to $T$,

$$
\begin{aligned}
& \frac{\mathrm{d}}{\mathrm{d} T}(\mathrm{TC})=-S_{C}+\left(H_{C}+\mu D_{C}\right) \\
& {\left[\begin{array}{l}
\frac{1}{R^{2} \mu(R+\mu)}\left[\begin{array}{l}
T R^{2}\left\{(a+b T)\left((R+\mu) e^{(R+\mu) T}-\operatorname{Re}^{R T}\right)+b\left(e^{(R+\mu) T}-e^{R T}\right)\right\} \\
-R^{2}(a+b T)\left(e^{(R+\mu) T}-e^{R T}\right)+R b \mu T e^{R T}-b \mu\left(e^{R T}-1\right) \\
-R^{2} a \mu T e^{R T}-R a \mu\left(1-e^{R T}\right)-R^{2} b \mu e^{R T}
\end{array}\right] \\
+\frac{b}{R \mu(R+\mu)^{2}}\left[R \mu T e^{R T}-\mu\left(e^{R T}-1\right)+R T\left(\operatorname{Re}^{R T}-(R+\mu) e^{(R+\mu) T}\right)+R\left(e^{(R+\mu) T}-e^{R T}\right)\right]
\end{array}\right]=0 .}
\end{aligned}
$$

That is, it can be written as

$$
\left[\begin{array}{l}
\frac{1}{R^{2} \mu(R+\mu)}\left[\begin{array}{l}
R^{2}(a+b T) T\left((R+\mu) e^{(R+\mu) T}-\operatorname{Re}^{R T}\right)+R^{2} b T\left(e^{(R+\mu) T}-e^{R T}\right) \\
-R^{2}(a+b T)\left(e^{(R+\mu) T}-e^{R T}\right)+R b \mu T e^{R T}-b \mu\left(e^{R T}-1\right) \\
-R^{2} a \mu T e^{R T}-R a \mu\left(1-e^{R T}\right)-R^{2} b \mu T^{2} e^{R T}
\end{array}\right] \\
+\frac{b}{R \mu(R+\mu)^{2}}\left[\begin{array}{l}
R \mu T e^{R T}-\mu\left(e^{R T}-1\right)+R^{2} T e^{R T}-R(R+\mu) T e^{(R+\mu) T} \\
-R\left(e^{R T}-e^{(R+\mu) T}\right)
\end{array}\right]
\end{array}\right]=\frac{S_{C}}{H_{C}+\mu D_{C}} .
$$

This is the optimum solution of $T$ in a higher-order equation. This equation can be evaluated by using Matlab. But for the reader's convenience; the equation is reduced to a fourth-order equation then a third-order equation in $T$. Expansion of exponential demand is evaluated up to fourth-order equation and simplifying them we can get,

$$
\left[\begin{array}{c}
\frac{1}{R+\mu}\left[\begin{array}{l}
\frac{R a T^{2}}{2}+\frac{a \mu T^{2}}{2}+\frac{b T^{2}}{2}+\frac{4 R b T^{3}}{3}+b \mu T^{3}+\frac{2 R^{2} a T^{3}}{3}+R a \mu T^{3} \\
+\frac{a \mu^{2} T^{3}}{3}+\frac{9 R^{2} b T^{4}}{8}+\frac{3 R b \mu T^{4}}{2}+\frac{b \mu^{2} T^{4}}{2}+\frac{R^{3} a T^{4}}{2}+\frac{3 R^{2} a \mu T^{4}}{4} \\
+\frac{R a \mu^{2} T^{4}}{2}+\frac{a \mu^{3} T^{4}}{8}-\frac{R^{2} a T^{4}}{8}
\end{array}\right] \\
+\frac{b}{(R+\mu)^{2}}\left[\begin{array}{l}
-\frac{R T^{2}}{2}-\frac{\mu T^{2}}{2}-\frac{2 R^{2} T^{3}}{3}-R \mu T^{3}-\frac{\mu^{2} T^{3}}{3}-\frac{3 R^{3} T^{4}}{8} \\
-\frac{3 R^{2} \mu T^{4}}{4}-\frac{R \mu^{2} T^{4}}{2}-\frac{\mu^{3} T^{4}}{8}
\end{array}\right]
\end{array}\right]=\frac{S_{C}}{H_{C}+\mu D_{C}} .
$$

After simplifications, the equation can be written in the following form

$$
\left[\begin{array}{l}
((R+\mu)(R a+a \mu+b)-b(R+\mu)) \frac{T^{2}}{2} \\
+\left[(R+\mu)\left(4 R b+3 b \mu+2 R^{2} a+3 R a \mu+a \mu^{2}\right)+b\left(-2 R^{2}-3 R \mu-\mu^{2}\right)\right] \frac{T^{3}}{3} \\
+\left[(R+\mu)\left(\begin{array}{c}
9 R^{2} b+12 R b \mu+4 b \mu^{2}+4 R^{3} a \\
+6 R^{2} a \mu+4 R a \mu^{2}+a \mu^{3}-R^{2} a
\end{array}\right)-b\left(\begin{array}{c}
3 R^{3}+6 R^{2} \mu \\
+4 R \mu^{2}+\mu^{3}
\end{array}\right)\right] \frac{T^{4}}{8}
\end{array}\right]=\frac{(R+\mu)^{2} S_{C} .}{H_{C}+\mu D_{C}} .
$$


TABLE 3. Variation of rate of deteriorating items with inventory and total cost with linear integrated with continuous compound demand.

\begin{tabular}{lllllll}
\hline \hline$\mu$ & $T$ & $Q$ & Setup cost & Holding cost & Deteriorating cost & Total cost \\
\hline 0.01 & 0.0879 & 457.02 & 1706.04 & 1515.40 & 108.24 & 3329.73 \\
0.02 & 0.0852 & 442.73 & 1759.72 & 1466.80 & 209.54 & 3436.07 \\
0.03 & 0.0827 & 428.84 & 1811.75 & 1422.60 & 304.84 & 3539.19 \\
0.04 & 0.0805 & 416.15 & 1862.28 & 1382.18 & 394.90 & 3639.37 \\
0.05 & 0.0784 & 404.51 & 1911.42 & 1345.03 & 480.36 & 3736.82 \\
0.06 & 0.0765 & 393.79 & 1959.30 & 1310.73 & 561.74 & 3831.77 \\
0.07 & 0.0747 & 383.87 & 2006.00 & 1278.93 & 639.46 & 4014.88 \\
0.08 & 0.0731 & 374.65 & 2051.65 & 1249.35 & 713.91 & 4103.34 \\
0.09 & 0.0715 & 366.05 & 2096.19 & 1221.74 & 785.40 & 4189.91 \\
0.10 & 0.0701 & 358.02 & 2139.83 & 1195.88 & 854.20 & \\
\hline
\end{tabular}

The above equation is in the fourth-order equation, then it is reduced to a third-order equation as follows,

$$
\begin{aligned}
(R+\mu)\left[2 b(R+\mu)+2 R^{2} a+3 R a \mu+a \mu^{2}\right] \frac{T^{3}}{3}+3 a(R+\mu) T^{2} & =\frac{6(R+\mu) S_{C}}{H_{C}+\mu D_{C}} \\
2\left[2 b(R+\mu)+2 R^{2} a+3 R a \mu+a \mu^{2}\right] T^{3}+3 a(R+\mu) T^{2} & =\frac{6(R+\mu) S_{C}}{H_{C}+\mu D_{C}} .
\end{aligned}
$$

which is an optimal solution in the third-order equation and it is solved based on the visual basic 6.0 program for generating the data.

Numerical example: let us consider the cost parameters

$$
Y=5000 \text { units, } H_{C}=7, D_{C}=50, S_{C}=150, \mu=0.01, R=0.1, a=4650, b=3985 .
$$

\section{Optimum solution}

The cubic equation for the given data in example,

$$
1968.23 T^{3}+1534.50 T^{2}-13.20=0 .
$$

The one positive real and two negative real roots of this third order equation is $T=0.08792,-0.09928$ and -0.76827 . The positive root $T=0.0879$ is considered in this model. Therefore, Cycle time $=0.0879$, Optimum Quantity $Q^{*}=457.02$, Setup cost $=1706.04$, Holding cost $=1515.40$, Deteriorating cost $=108.24$, Total cost $=$ 3329.73.

From the above Table 3, it is observed that a study of the rate of the deteriorative items with cycle time, optimum quantity, setup cost, holding cost, deteriorating cost, and total cost. There is a positive relationship between the increase in the rate of deterioration for items $\mu$ and setup costs, deteriorating costs, and total costs, while there is a negative relationship between the increase in the rate of deterioration for items $\mu$ and cycle time, optimum quantity, and holding cost.

The graphical representations between total cost with rate of deteriorative items is given in Figure 3 :

\section{Sensitivity analysis}

Managerial insights: a sensitivity analysis is performed to study the effects of changes in the system parameters, rate of deteriorating items $\mu$, ordering cost per order $\left(S_{C}\right)$, holding cost per unit time $\left(H_{C}\right)$, deteriorating cost per unit time $\left(D_{C}\right)$, and rate of increase in demand $(R)$ on optimal values that is optimal cycle time $(T)$, optimal quantity $(Q)$, setup cost, holding cost, deteriorating cost and total cost. The sensitivity analysis 


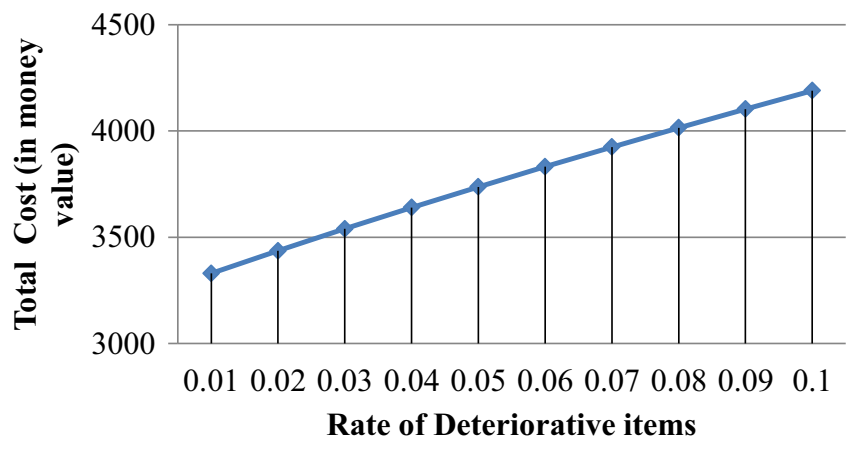

FiguRE 3. Relationship between rate of deteriorative items with total cost.

is performed by changing (increasing or decreasing) the parameter taking at a time, keeping the remaining parameters at their original values. The following influences can be obtained from sensitivity analysis based on Table 4 .

(1) There is a positive relationship between the increase in the setup costs per set $\left(S_{C}\right)$ and the cycle time, the optimum quantity, setup costs, holding costs, deteriorating costs, and total costs.

(2) There is a positive relationship between the increase in the holding cost per unit time $\left(H_{C}\right)$ and the setup cost, holding cost, and total cost while there is a negative relationship between the increase in the holding cost per unit time $\left(H_{C}\right)$ and the cycle time, optimal quantity and deteriorating cost.

(3) There is a positive relationship between the increase in the rate of demand $(R)$ and the setup cost, holding cost, deteriorative cost and total cost, while there is a negative relationship between the increase in the rate of demand $(R)$ and the cycle time, and optimum quantity.

(4) Similarly, other parameters, cost of deteriorating item per unit $\left(D_{C}\right), a$, and $b$ can also be observed from Table 4

\subsection{EOQ inventory model for deteriorating items with integrated quadratic and continuous compound demand}

This model has developed a deteriorating inventory model in which demand is quadratically integrated with continuous compound demand which is a function of time that is $Y=\left(a+b t+c t^{2}\right) e^{R t}$, where $R>0$ at time $t$ and "R" stands for the increase in demand, "a" stands for constant that is initial demand and "b" and "c" are demand-based time. Let $Q$ be the units of item arrive at the inventory system at the beginning of each cycle. The inventory level decreases due to demand and deteriorating till it becomes zero in the interval $(0, T)$. The total process is repeated. The differential equation describing the instantaneous states of $I(t)$ in the interval $[0, T]$ is given below:

$$
\frac{\mathrm{d}}{\mathrm{d} t} I(t)+\mu I(t)=-\left(a+b t+c t^{2}\right) e^{R t} ; \quad 0<t<T_{1}
$$

with the boundary conditions

$$
I(0)=Q, \quad I(T)=0 .
$$

From the differential equation (4.9)

$$
I(t)=\left[\begin{array}{l}
\frac{1}{R+\mu}\left[\left(a+b T+c T^{2}\right) e^{(R+\mu) T-\mu t}-\left(a+b t+c t^{2}\right) e^{R t}\right] \\
+\frac{1}{(R+\mu)^{2}}\left[(b+2 c t) e^{R t}-(b+2 c T) e^{(R+\mu) T-\mu t}\right]+\frac{2 c}{(R+\mu)^{3}}\left[\left(e^{(R+\mu) T-\mu t}-e^{R t}\right)\right]
\end{array}\right] .
$$


TABLE 4. Effect of demand and cost parameters on optimal values and linear with integrated continuous compound demand.

\begin{tabular}{|c|c|c|c|c|c|c|c|}
\hline \multirow{2}{*}{\multicolumn{2}{|c|}{ Cost parameters }} & \multicolumn{6}{|c|}{ Optimal values } \\
\hline & & $T$ & $Q$ & Setup cost & Holding cost & Deteriorative cost & Total cost \\
\hline \multirow{5}{*}{$S_{C}$} & 130 & 0.0821 & 425.82 & 1582.92 & 1410.32 & 100.73 & 3093.99 \\
\hline & 140 & 0.0851 & 439.74 & 1645.49 & 1463.81 & 104.55 & 3213.86 \\
\hline & 150 & 0.0879 & 457.02 & 1706.04 & 1515.40 & 108.24 & 3329.73 \\
\hline & 160 & 0.0906 & 470.80 & 1764.77 & 1565.38 & 111.81 & 3441.97 \\
\hline & 170 & 0.0933 & 487.18 & 1821.85 & 1613.81 & 115.27 & 3550.94 \\
\hline \multirow{5}{*}{$H_{C}$} & 5 & 0.1018 & 532.64 & 1472.65 & 1265.08 & 126.51 & 2864.25 \\
\hline & 6 & 0.0941 & 492.81 & 1593.89 & 1395.79 & 116.31 & 3106.01 \\
\hline & 7 & 0.0879 & 457.02 & 1706.04 & 1515.40 & 108.24 & 3329.73 \\
\hline & 8 & 0.0828 & 431.65 & 1810.89 & 1626.39 & 101.64 & 3538.93 \\
\hline & 9 & 0.0785 & 405.04 & 1909.69 & 1730.29 & 96.12 & 3736.11 \\
\hline \multirow{5}{*}{$D_{C}$} & 30 & 0.0890 & 463.16 & 1684.25 & 1536.15 & 65.83 & 3286.24 \\
\hline & 40 & 0.0884 & 460.26 & 1695.18 & 1525.69 & 87.18 & 3308.06 \\
\hline & 50 & 0.0879 & 457.02 & 1706.04 & 1515.40 & 108.24 & 3329.73 \\
\hline & 60 & 0.0873 & 454.24 & 1716.83 & 1505.38 & 129.03 & 3351.25 \\
\hline & 70 & 0.0868 & 450.93 & 1727.55 & 1495.53 & 149.55 & 3372.64 \\
\hline \multirow{5}{*}{$a$} & 4350 & 0.0904 & 443.56 & 1657.79 & 1466.20 & 104.72 & 3228.72 \\
\hline & 4500 & 0.0891 & 451.25 & 1682.07 & 1491.02 & 106.50 & 3279.59 \\
\hline & 4650 & 0.0879 & 457.02 & 1706.04 & 1515.40 & 108.24 & 3329.73 \\
\hline & 4800 & 0.0867 & 464.03 & 1729.72 & 1539.47 & 109.96 & 3379.16 \\
\hline & 4950 & 0.0855 & 469.67 & 1753.12 & 1563.14 & 111.65 & 3427.92 \\
\hline \multirow{5}{*}{$b$} & 3685 & 0.0882 & 455.17 & 1700.50 & 1515.20 & 108.22 & 3323.94 \\
\hline & 3835 & 0.0880 & 456.14 & 1703.28 & 1515.32 & 108.23 & 3326.84 \\
\hline & 3985 & 0.0879 & 457.02 & 1706.04 & 1515.40 & 108.24 & 3329.73 \\
\hline & 4135 & 0.0877 & 458.04 & 1708.80 & 1515.55 & 108.25 & 3332.61 \\
\hline & 4285 & 0.0876 & 458.91 & 1711.54 & 1515.68 & 108.26 & 3335.48 \\
\hline \multirow{9}{*}{$R$} & 0.10 & 0.0879 & 457.02 & 1706.04 & 1515.40 & 108.24 & 3329.73 \\
\hline & 0.15 & 0.0877 & 456.73 & 1710.31 & 1517.69 & 108.40 & 3336.41 \\
\hline & 0.20 & 0.0874 & 456.37 & 1714.54 & 1519.95 & 108.56 & 3343.06 \\
\hline & 0.25 & 0.0872 & 456.02 & 1718.74 & 1522.19 & 108.72 & 3349.66 \\
\hline & 0.30 & 0.0870 & 455.68 & 1722.91 & 1524.43 & 108.88 & 3356.23 \\
\hline & 0.35 & 0.0868 & 455.34 & 1727.05 & 1526.65 & 109.04 & 3362.75 \\
\hline & 0.40 & 0.0866 & 455.01 & 1731.17 & 1528.86 & 109.20 & 3369.24 \\
\hline & 0.45 & 0.0864 & 454.68 & 1735.26 & 1531.05 & 109.36 & 3375.68 \\
\hline & 0.50 & 0.0862 & 454.36 & 1739.32 & 1533.24 & 109.51 & 3382.08 \\
\hline
\end{tabular}

\section{Note:}

(i) When $a=Y$ and $b=c=0$, then $I(t)=\frac{Y}{R+\mu}\left[e^{(R+\mu) T-\mu t}-e^{R t}\right]$ which is continuous compound demand. (As per Model 1)

(ii) When $R=0$, then

$$
I(t)=\left[\left(\frac{a}{\mu}+\frac{b T}{\mu}+\frac{c T^{2}}{\mu}\right)-\left(\frac{b}{\mu^{2}}+\frac{2 c T}{\mu^{2}}\right)+\frac{2 c}{\mu^{3}}\right] e^{(T-t) \mu}-\left(\frac{a}{\mu}+\frac{b t}{\mu}+\frac{c t^{2}}{\mu}\right)+\left(\frac{b}{\mu^{2}}+\frac{2 c t}{\mu^{2}}\right)-\frac{2 c}{\mu^{3}}
$$

which is Quadratic Demand.

(iii) When $a=D$ and $b=0$ and $R=0$, then $\left.I(t)=\frac{D}{\mu}\left(e^{(T-t) \mu}-1\right)\right)$ constant Demand. 
(iv) When $c=0$ in (ii), then $I(t)=\left(\frac{a}{\mu}+\frac{b T}{\mu}-\frac{b}{\mu^{2}}\right) e^{(T-t) \mu}-\left(\frac{a}{\mu}+\frac{b t}{\mu}-\frac{b}{\mu^{2}}\right)$

which is linear demand.

(Appendix B)

\section{To find Q:}

$$
I(0)=Q \Rightarrow Q=\left[\begin{array}{l}
\frac{1}{R+\mu}\left[\left(a+b T+c T^{2}\right) e^{(R+\mu) T}-a\right] \\
+\frac{1}{(R+\mu)^{2}}\left[b-(b+2 c T) e^{(R+\mu) T}\right]+\frac{2 c}{(R+\mu)^{3}}\left[\left(e^{(R+\mu) T}-1\right)\right]
\end{array}\right] .
$$

On simplification,

$$
Q=a T+\frac{a(R+\mu) T^{2}}{2}+\frac{a(R+\mu)^{2} T^{3}}{6}+\frac{b T^{2}}{2}+\frac{b(R+\mu) T^{3}}{3}+\frac{C T^{3}}{3} .
$$

Total cost $\mathbf{T C}(T)$ : Total cost comprised of the sum of the setup cost, holding cost, and deteriorating cost. They are grouped after evaluating the above cost individually.

Total cost $(\mathrm{TC})=$ Setup cost + holding cost + deteriorating cost.

$$
\text { Setup cost }=\frac{S_{C}}{T} .
$$

(2) Holding cost $=\frac{H_{C}}{T}\left[\int_{0}^{T}\left(\begin{array}{l}\frac{1}{R+\mu}\left[\left(a+b T+c T^{2}\right) e^{(R+\mu) T-\mu t}-\left(a+b t+c t^{2}\right) e^{R t}\right] \\ +\frac{1}{(R+\mu)^{2}}\left[(b+2 c t) e^{R t}-(b+2 c T) e^{(R+\mu) T-\mu t}\right] \\ +\frac{2 c}{(R+\mu)^{3}}\left(e^{(R+\mu) T-\mu t}-e^{R t}\right)\end{array}\right]\right]$

On further simplifications,

$$
\begin{gathered}
=\frac{H_{C}}{T}\left[\begin{array}{l}
\frac{1}{R^{3} \mu(R+\mu)}\left(\begin{array}{l}
-R^{3}\left(a+b T+c T^{2}\right) e^{R T}-R^{2} \mu\left(a+b T+c T^{2}\right) e^{R T} \\
+R \mu(b+2 c T) e^{R T}-2 c \mu e^{R T}+R^{3}\left(a+b T+c T^{2}\right) e^{(R+\mu) T} \\
-R^{2} a \mu-R b \mu+2 c \mu
\end{array}\right) \\
+\frac{1}{R^{2} \mu(R+\mu)^{2}}\left[\begin{array}{l}
R \mu(b+2 c T) e^{R T}-2 c \mu e^{R t}+R^{2}(b+2 c T) e^{R T} \\
-R \mu b+2 c \mu-R^{2}(b+2 c T) e^{(R+\mu) T}
\end{array}\right] \\
\frac{-2 c}{R \mu(R+\mu)^{3}}\left[\begin{array}{l}
\operatorname{Re}^{R T}+\mu e^{R T}-\operatorname{Re}^{(R+\mu) T}-\mu
\end{array}\right]
\end{array}\right) \\
=\frac{H_{C}}{T}\left[\begin{array}{l}
\frac{1}{R^{3} \mu(R+\mu)}\left(\begin{array}{l}
-R^{3}\left(a+b T+c T^{2}\right)\left(e^{(R+\mu) T}-e^{R T}\right)+R^{2} a \mu\left(1-e^{R T}\right) \\
-R^{2} \mu\left(b T+c T^{2}\right) e^{R T}+R b \mu\left(e^{R T}-1\right)+2 R c \mu T e^{R T}+2 c \mu\left(1-e^{R T}\right)
\end{array}\right) \\
+\frac{1}{R^{2} \mu(R+\mu)^{2}}\left(\begin{array}{l}
R^{2}(b+2 c T)\left(e^{R T}-e^{(R+\mu) T}\right)+b R \mu\left(e^{R T}-1\right) \\
+2 R c \mu T e^{R T}+2 c \mu\left(1-e^{R T}\right)
\end{array}\right) \\
\frac{-2 c}{R \mu(R+\mu)^{3}}\left(R\left(e^{R T}-e^{(R+\mu) T}\right)+\mu\left(e^{R T}-1\right)\right)
\end{array}\right] .
\end{gathered}
$$

(3) Deteriorative cost

$$
=\frac{\mu D_{C}}{T}\left[\begin{array}{l}
\frac{1}{R^{3} \mu(R+\mu)}\left(\begin{array}{l}
-R^{3}\left(a+b T+c T^{2}\right)\left(e^{(R+\mu) T}-e^{R T}\right)+R^{2} a \mu\left(1-e^{R T}\right) \\
-R^{2} \mu\left(b T+c T^{2}\right) e^{R T}+R b \mu\left(e^{R T}-1\right)+2 R c \mu T e^{R T}+2 c \mu\left(1-e^{R T}\right)
\end{array}\right) \\
+\frac{1}{R^{2} \mu(R+\mu)^{2}}\left(\begin{array}{l}
R^{2}(b+2 c T)\left(e^{R T}-e^{(R+\mu) T}\right)+b R \mu\left(e^{R T}-1\right) \\
+2 R c \mu T e^{R T}+2 c \mu\left(1-e^{R T}\right)
\end{array}\right) \\
\frac{-2 c}{R \mu(R+\mu)^{3}}\left(R\left(e^{R T}-e^{(R+\mu) T}\right)+\mu\left(e^{R T}-1\right)\right)
\end{array}\right] .
$$


Therefore, the total cost

$$
\mathrm{TC}=\frac{S_{C}}{T}+\frac{H_{C}+\mu D_{C}}{T}\left[\begin{array}{c}
\frac{1}{R^{3} \mu(R+\mu)}\left(\begin{array}{l}
R^{3}\left(a+b T+c T^{2}\right)\left(e^{(R+\mu) T}-e^{R T}\right)+R^{2} a \mu\left(1-e^{R T}\right) \\
-R^{2} \mu\left(b T+c T^{2}\right) e^{R T}+R b \mu\left(e^{R T}-1\right)+2 R c \mu T e^{R T} \\
+2 c \mu\left(1-e^{R T}\right)
\end{array}\right) \\
+\frac{1}{R^{2} \mu(R+\mu)^{2}}\left(\begin{array}{l}
R^{2}(b+2 c T)\left(e^{R T}-e^{(R+\mu) T}\right)+b R \mu\left(e^{R T}-1\right) \\
+2 R c \mu T e^{R T}+2 c \mu\left(1-e^{R T}\right) \\
\frac{-2 c}{R \mu(R+\mu)^{3}}\left(R\left(e^{R T}-e^{(R+\mu) T}\right)+\mu\left(e^{R T}-1\right)\right)
\end{array}\right)
\end{array}\right] .
$$

\section{Optimality conditions:}

$$
\frac{\mathrm{d}}{\mathrm{d} T} \mathrm{TC}(T)=0 \quad \text { and } \quad \frac{\mathrm{d}^{2}}{\mathrm{~d} T^{2}} \mathrm{TC}(T)>0
$$

Differentiate the equation (4.24) with respect to $T$

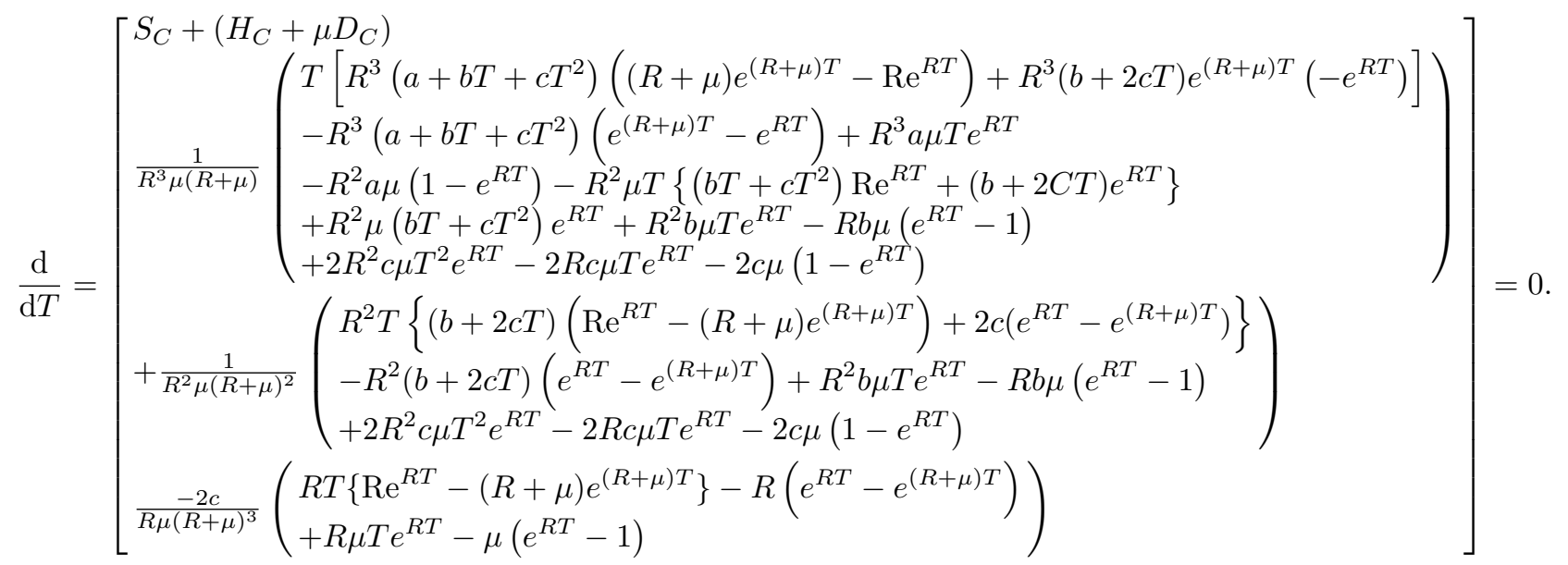

This should be equal to

$$
\left(\begin{array}{c}
\frac{1}{R^{3} \mu(R+\mu)}\left(\begin{array}{l}
R^{3} T\left(a+b T+c T^{2}\right)\left((R+\mu) e^{(R+\mu) T}-\mathrm{Re}^{R T}\right)+R^{3} T(b+2 c T) \\
\left(e^{(R+\mu) T}-e^{R T}\right)-R^{3}\left(a+b T+c T^{2}\right)\left(e^{(R+\mu) T}-e^{R T}\right) \\
-R^{3} a \mu T e^{R T}-R^{2} a \mu\left(1-e^{R T}\right)-R^{3} \mu T\left(b T+c T^{2}\right) e^{R T} \\
-R^{2} \mu T(b+2 c T) e^{R T}+R^{2} \mu\left(b T+c T^{2}\right) e^{R T}+R^{2} b \mu T e^{R T} \\
-R b \mu\left(e^{R T}-1\right)+2 R^{2} c \mu T^{2} e^{R T}-2 R c \mu T e^{R T}-2 c \mu\left(1-e^{R T}\right)
\end{array}\right) \\
+\frac{1}{R^{2} \mu(R+\mu)^{2}}\left(\begin{array}{l}
R^{2}(b+2 c T) T\left(\operatorname{Re}^{R T}-(R+\mu) e^{(R+\mu) T}\right)+2 R^{2} C T\left(e^{R T}-e^{(R+\mu) T}\right) \\
-R^{2}(b+2 c T)\left(e^{R T}-e^{(R+\mu) T}\right)+R^{2} b \mu T e^{R T}-R b \mu\left(e^{R T}-1\right) \\
+2 R^{2} c \mu T^{2} e^{R T}-2 R c \mu T e^{R T}-2 c \mu\left(1-e^{R T}\right)
\end{array}\right) \\
\frac{-2 c}{R \mu(R+\mu)^{3}}\left(\begin{array}{l}
R T\left(R^{R T}-(R+\mu) e^{(R+\mu) T}\right)-R\left(e^{R T}-e^{(R+\mu) T}\right) \\
+R \mu T e^{R T}-\mu\left(e^{R T}-1\right)
\end{array}\right)=\frac{S_{C}}{H_{C}+\mu D_{C}}
\end{array}\right) .
$$

The above equation is the optimum solution in a higher-order equation and it is solved by using Mat lab. But, for the reader's convenience, the above equation is reduced to forth order equation and it is solved by using 
Visual Basic 6.0. On simplification, the fourth-order equation is

$$
\begin{gathered}
\left(\begin{array}{c}
102 R^{2} c \mu+18 R^{4} b+33 R^{3} b \mu+43 R^{2} b \mu^{2}+9 R^{5} a+36 R^{4} a \mu \\
+57 R^{3} a \mu^{2}+45 R^{2} a \mu^{3}+102 R c \mu^{2}+37 R b \mu^{3}+18 R a \mu^{4} \\
+18 c \mu^{3}+9 b \mu^{4}+3 a \mu^{5}-32 R^{2} c \mu^{2}-32 R c \mu^{3}+18 R^{3} c
\end{array}\right) T^{4} \\
+8\left(\begin{array}{l}
2 R^{3} b+9 R^{2} b \mu+2 R^{4} a+7 R^{3} a \mu+9 R^{2} a \mu^{2}+6 R b \mu^{2} \\
+5 R a \mu^{2}+2 b \mu^{3}+a \mu^{4}-5 R^{2} b \mu^{2}-2 R b \mu^{3}
\end{array}\right) T^{3} \\
+12 a(R+\mu)^{3} T^{2}=\frac{24(R+\mu)^{3} S_{C}}{H_{C}+\mu D_{C}}
\end{gathered}
$$

which is the optimum solution for $T$ in the fourth-order equation.

\section{Note:}

(1) When $R=0$

$$
3\left(a \mu^{2}+3 b \mu+6 c\right) T^{4}+8(a \mu+2 b) T^{3}+12 a T^{2}=\frac{24 S_{C}}{H_{C}+\mu D_{C}}
$$

which is quadratic demand.

(Appendix C)

(2) When $R=0 ; c=0$

$$
3 \mu(a \mu+3 b) T^{4}+8(a \mu+2 b) T^{3}+12 a^{2}=\frac{24 S_{C}}{H_{C}+\mu D_{C}}
$$

which is a linear demand.

(Appendix B)

(3) When $a=Y ; b=c=0$

$$
3\left(3 R^{3}+6 R^{2} \mu+4 R \mu^{2}+\mu^{3}\right) T^{4}+8\left(2 R^{2}+3 R \mu+\mu^{2}\right) T^{3}+12(R+\mu) T^{2}=\frac{24(R+\mu) S_{C}}{D\left(H_{C}+\mu D_{C}\right)}
$$

which is CCD. (As per model 1)

(4) When the higher-order equation equal to zero, then $T=\sqrt{\frac{2 S_{C}}{Y\left(H_{C}+\mu D_{C}\right)}}$

which is standard inventory models.

(Appendix A)

Numerical example: let us consider the cost parameters

$$
Y=5000 \text { units, } H_{C}=7, D_{C}=50, S_{C}=150, \mu=0.01, R=0.1, a=4000, b=3700, c=2400 \text {. }
$$

\section{Optimum solution}

The forth order equation is $T^{4}+1.2266 T^{3}+0.9046 T^{2}-0.0078=0$, in which the roots are one positive real root, one negative real root and two complex roots. The positive root $T=0.0878$ is considered in this model. Therefore, Cycle time $=0.0878$, Optimum Quantity $Q^{*}=420.71$, Setup cost $=1708.12$, Holding cost $=1132.20$, Deteriorating cost $=80.87$, Total cost $=2921.19$.

The above Table 5, a study on the rate of the deteriorative items with cycle time, optimum quantity, setup cost, holding cost, deteriorating cost and total cost are observed. There is a positive relationship between the increase in the rate of deterioration for items $\mu$ and setup costs, holding cost, deteriorating costs, and total costs, while there is a negative relationship between the increase in the rate of deterioration for items $\mu$ and cycle time, and optimum quantity. 
TABLE 5. Variation of rate of deteriorating items with inventory and total cost.

\begin{tabular}{lllllll}
\hline \hline$\mu$ & $T$ & $Q$ & Setup cost & Holding cost & Deteriorating cost & Total cost \\
\hline 0.01 & 0.0878 & 420.71 & 1708.12 & 1132.20 & 80.87 & 2921.19 \\
0.02 & 0.0848 & 405.86 & 1768.82 & 1156.22 & 165.17 & 3090.22 \\
0.03 & 0.0821 & 392.80 & 1826.04 & 1165.51 & 249.75 & 3241.32 \\
0.04 & 0.0797 & 381.18 & 1880.34 & 1165.65 & 333.04 & 3379.04 \\
0.05 & 0.0776 & 370.72 & 1932.14 & 1160.01 & 414.29 & 3506.45 \\
0.06 & 0.0756 & 361.21 & 1981.82 & 1150.71 & 493.16 & 3625.69 \\
0.07 & 0.0739 & 352.52 & 2029.67 & 1139.08 & 569.54 & 3738.29 \\
0.08 & 0.0722 & 344.51 & 2075.90 & 1126.03 & 643.44 & 3845.37 \\
0.09 & 0.0707 & 337.08 & 2120.71 & 1112.13 & 714.94 & 3947.79 \\
0.10 & 0.0693 & 330.17 & 2164.26 & 1097.80 & 784.14 & 4046.22 \\
\hline
\end{tabular}

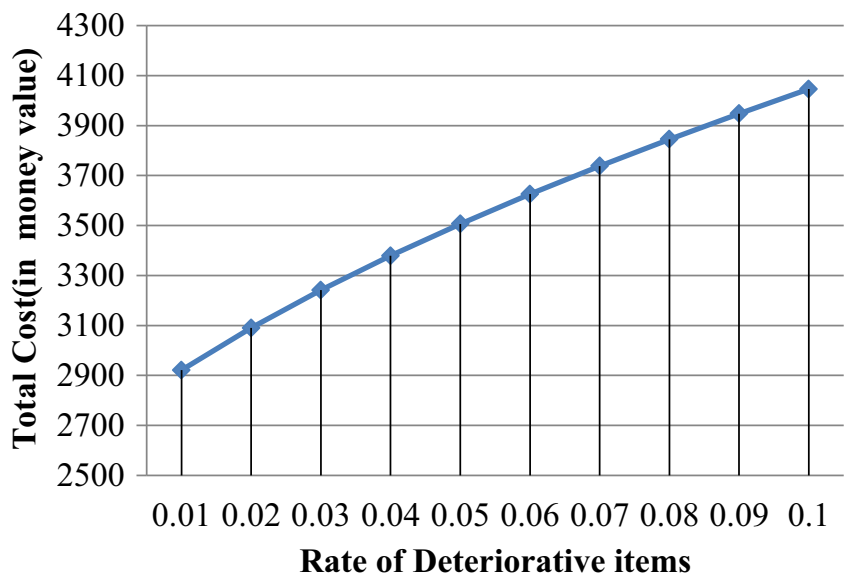

FIGURE 4. Relationship between rate of deteriorative items and total cost in quadratic integrated with continuous compound demand.

The graphical representations between total cost with rate of deteriorative items is given in Figure 4:

\section{Sensitivity analysis:}

Managerial insights: a sensitivity analysis is performed to study the effects of changes in the system parameters, rate of deteriorating items $\mu$, ordering cost per order $\left(S_{C}\right)$, holding cost per unit time $\left(H_{C}\right)$, deteriorating cost per unit time $\left(D_{C}\right)$, and rate of increase in demand $(R)$ on optimal values that is optimal cycle time $(T)$, optimal quantity $(Q)$, setup cost, holding cost, deteriorating cost and total cost. The sensitivity analysis is performed by changing (increasing or decreasing) the parameter taking at a time, keeping the remaining parameters at their original values. The following influences are obtained from sensitivity analysis based on Table 6.

(1) There is a positive relationship between the increase in the setup costs per set $\left(S_{C}\right)$ and the cycle time, the optimum quantity, setup costs, holding costs, deteriorating costs, and total costs.

(2) There is a positive relationship between the increase in the holding cost per unit time $\left(H_{C}\right)$ and the setup cost, holding cost, and total cost while there is a negative relationship between the increase in the holding cost per unit time $\left(H_{C}\right)$ and the cycle time, optimal quantity and deteriorating cost. 
TABLE 6. Effect of Demand and cost parameters on optimal values in quadratic integrated with continuous compound demand.

\begin{tabular}{|c|c|c|c|c|c|c|c|}
\hline \multirow{2}{*}{\multicolumn{2}{|c|}{ Cost parameters }} & \multicolumn{6}{|c|}{ Optimal values } \\
\hline & & $T$ & $Q$ & Setup cost & Holding cost & Deteriorative cost & Total cost \\
\hline \multirow{5}{*}{$S_{C}$} & 130 & 0.0820 & 392.15 & 1583.91 & 1075.39 & 76.81 & 2736.12 \\
\hline & 140 & 0.0850 & 406.69 & 1647.01 & 1104.66 & 78.90 & 2830.57 \\
\hline & 150 & 0.0878 & 420.71 & 1708.12 & 1132.20 & 80.87 & 2921.19 \\
\hline & 160 & 0.0905 & 434.25 & 1767.43 & 1158.19 & 82.72 & 3008.35 \\
\hline & 170 & 0.0931 & 447.35 & 1825.10 & 1182.78 & 84.48 & 3092.37 \\
\hline \multirow{5}{*}{$H_{C}$} & 5 & 0.1015 & 489.81 & 1476.65 & 899.00 & 89.90 & 2465.56 \\
\hline & 6 & 0.0939 & 451.31 & 1596.87 & 1020.07 & 85.01 & 2701.95 \\
\hline & 7 & 0.0878 & 420.71 & 1708.12 & 1132.20 & 80.87 & 2921.19 \\
\hline & 8 & 0.0827 & 395.62 & 1812.14 & 1237.08 & 77.31 & 3126.54 \\
\hline & 9 & 0.0785 & 374.57 & 1910.19 & 1335.94 & 74.21 & 3320.36 \\
\hline \multirow{5}{*}{$D_{C}$} & 30 & 0.0889 & 426.33 & 1686.49 & 1143.06 & 48.98 & 2878.54 \\
\hline & 40 & 0.0883 & 423.49 & 1697.34 & 1137.58 & 65.01 & 2899.94 \\
\hline & 50 & 0.0878 & 420.71 & 1708.12 & 1132.20 & 80.87 & 2921.19 \\
\hline & 60 & 0.0872 & 417.98 & 1718.82 & 1126.89 & 96.59 & 2942.31 \\
\hline & 70 & 0.0867 & 415.31 & 1729.45 & 1121.65 & 112.16 & 2963.29 \\
\hline \multirow{5}{*}{$a$} & 4300 & 0.0903 & 406.10 & 1660.29 & 1061.00 & 75.78 & 2797.08 \\
\hline & 4450 & 0.0890 & 413.47 & 1684.35 & 1097.10 & 78.36 & 2859.81 \\
\hline & 4600 & 0.0878 & 420.71 & 1708.12 & 1132.20 & 80.87 & 2921.19 \\
\hline & 4750 & 0.0866 & 427.82 & 1731.61 & 1166.36 & 83.31 & 2981.28 \\
\hline & 4900 & 0.0854 & 434.82 & 1754.83 & 1199.64 & 85.68 & 3040.17 \\
\hline \multirow{5}{*}{$b$} & 3400 & 0.0881 & 421.12 & 1701.99 & 1129.82 & 80.70 & 2912.51 \\
\hline & 3550 & 0.0879 & 420.91 & 1705.06 & 1131.01 & 80.78 & 2916.86 \\
\hline & 3700 & 0.0878 & 420.71 & 1708.12 & 1132.20 & 80.87 & 2921.19 \\
\hline & 3850 & 0.0876 & 420.50 & 1711.16 & 1133.37 & 80.95 & 2925.49 \\
\hline & 4000 & 0.0875 & 420.31 & 1714.19 & 1134.54 & 81.03 & 2929.77 \\
\hline \multirow{5}{*}{$c$} & 2100 & 0.0878 & 420.81 & 1707.46 & 1177.17 & 84.08 & 2968.72 \\
\hline & 2250 & 0.0878 & 420.76 & 1707.79 & 1154.67 & 82.47 & 2944.95 \\
\hline & 2400 & 0.0878 & 420.71 & 1708.12 & 1132.20 & 80.87 & 2921.19 \\
\hline & 2550 & 0.0877 & 420.66 & 1708.44 & 1109.93 & 79.26 & 2897.45 \\
\hline & 2700 & 0.0877 & 420.61 & 1708.77 & 1087.29 & 77.66 & 2873.73 \\
\hline \multirow{10}{*}{$R$} & 0.05 & 0.0878 & 418.81 & 1712.06 & 884.34 & 63.16 & 2659.58 \\
\hline & 0.10 & 0.0877 & 420.71 & 1708.12 & 1132.20 & 80.87 & 2921.19 \\
\hline & 0.15 & 0.0876 & 421.25 & 1709.45 & 1239.36 & 88.52 & 3037.35 \\
\hline & 0.20 & 0.0875 & 421.39 & 1712.34 & 1298.59 & 92.75 & 3103.69 \\
\hline & 0.25 & 0.0874 & 421.36 & 1715.87 & 1336.06 & 95.43 & 3147.37 \\
\hline & 0.30 & 0.0872 & 421.25 & 1719.74 & 1361.86 & 97.27 & 3178.88 \\
\hline & 0.35 & 0.0870 & 421.08 & 1723.79 & 1380.69 & 98.62 & 3203.10 \\
\hline & 0.40 & 0.0868 & 420.88 & 1727.94 & 1395.01 & 99.64 & 3222.60 \\
\hline & 0.45 & 0.0865 & 420.66 & 1732.17 & 1406.25 & 100.44 & 3238.87 \\
\hline & 0.50 & 0.0863 & 420.43 & 1736.45 & 1515.30 & 101.09 & 3252.85 \\
\hline
\end{tabular}

(3) There is a positive relationship between the increase in the rate of demand $(R)$ and the optimum quantity, holding cost, deteriorative cost and total cost, while there is a negative relationship between the increase in the rate of demand $(R)$ and the cycle time, and setup cost.

(4) Similarly, other parameters, rate of deteriorating items $D_{C}$, initial demand "a" and demands based on time "b" and "c" can also be observed from Table 6. 
TABLE 7. Relationship between CCD, Linear with CCD and Quadratic with CCD.

\begin{tabular}{llll}
\hline \hline Item of cost & CCD & Linear with CCD & Quadratic with CCD \\
\hline Setup cost & 1687.45 & 1706.04 & 1708.12 \\
Holding cost & 1565.27 & 1515.40 & 1132.20 \\
Deteriorative cost & 111.80 & 108.24 & 80.87 \\
Total cost & 3364.53 & 3329.73 & 2921.19 \\
\hline
\end{tabular}
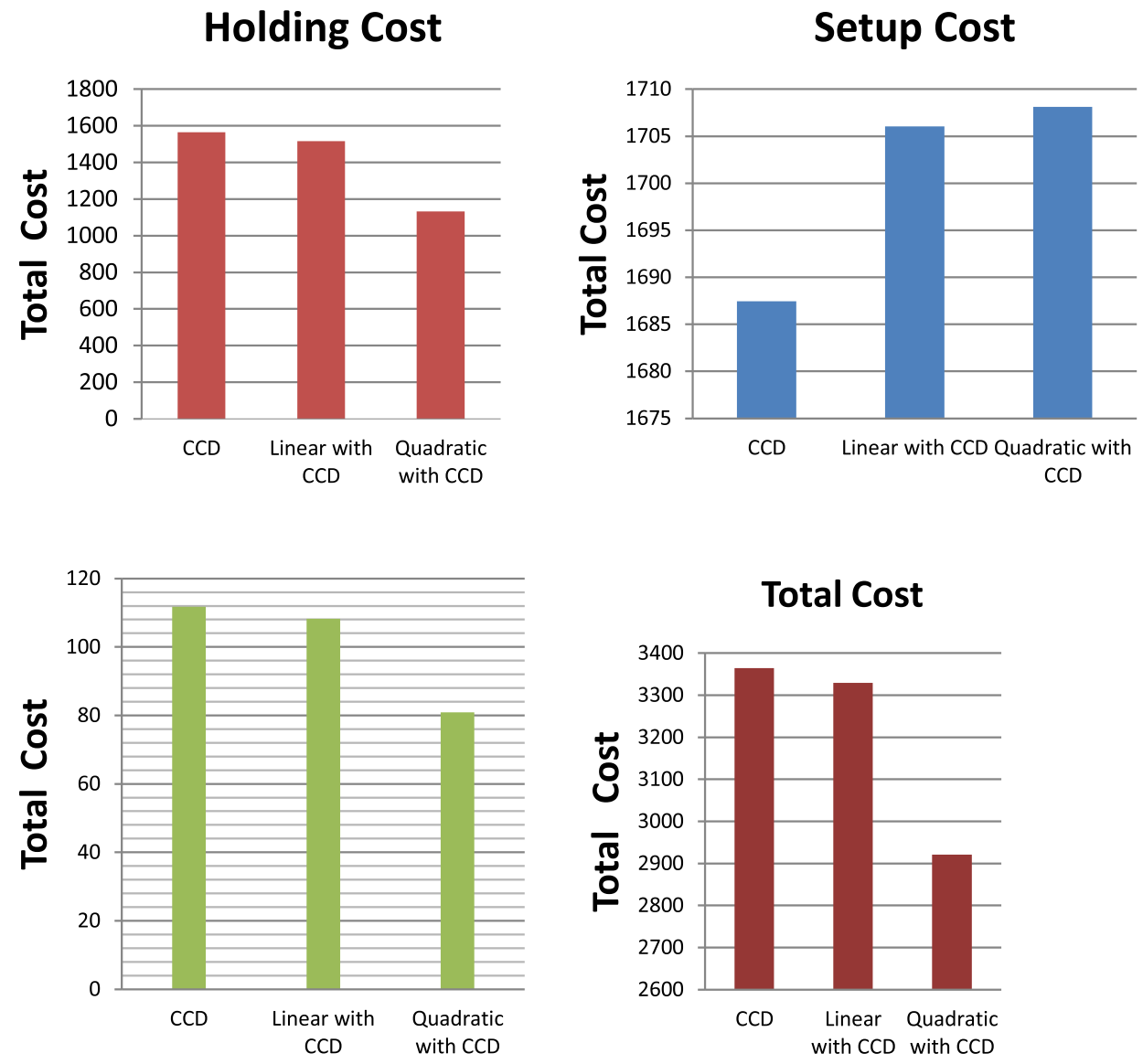

Figure 5. Relationship between constant demand and continuous compound demand.

\section{Comparative Study}

The relationship between continuous compound demand, linear demand integrated with continuous compound demand, and quadratic demand integrated with continuous compound demand is presented in the following table. From Table 7, it is observed that The setup cost is lower in constant demand than in linear CCD and quadratic CCD. The holding cost, deterioration cost, and total cost are lower for quadratic CCD compared to CDD and linear CCD (Fig. 5). 


\section{Conclusion}

This paper considers inventory models with integrated time-dependent demands in third-order equations. The setup cost is lower in constant demand than in linear CCD and quadratic CCD. The holding cost, deterioration cost, and total cost are lower for quadratic CCD compared to CDD and linear CCD. There is a positive relationship between the increase in the rate of deterioration for items $\mu$ and setup costs, deteriorating costs, and total costs, while there is a negative relationship between the increase in the rate of deterioration for items $\mu$ and cycle time, optimum quantity, and holding cost. There is a positive relationship between the increase in the setup costs per set $\left(S_{C}\right)$ and the cycle time, the optimum quantity, setup costs, holding costs, deteriorating costs, and total costs. There is a positive relationship between the increase in the holding cost per unit time $\left(H_{C}\right)$ and the setup cost, holding cost, and total cost while there is a negative relationship between the increase in the holding cost per unit time $\left(H_{C}\right)$ and the cycle time, optimal quantity and deteriorating cost. There is a positive relationship between the increase in the rate of demand $(R)$ and the setup cost and total cost, while there is a negative relationship between the increase in the rate of demand $(R)$ and the cycle time, optimum quantity, holding cost, and cost of deterioration. There is no reduction in cost sensitivity.

Several extensions that could be made to this research are:

(1) In this study of inventory models, demand is a continuous compound. The analysis of probabilistic demand can be another addition to this report.

(2) The Inventory models developed in this research are for a single period only. We can take the Inventory models with multiple items into consideration for further research.

(3) The developed inventory models in this study introduce only one concept at a time. In the future, models with the combination of several concepts may be used to determine the optimal policies.

(4) The proposed model can assist the manufacturer and retailer in accurately determining the optimal quantity, cycle time, and inventory total cost. Moreover, the proposed inventory model can be used in inventory control of certain items such as food items, fashionable commodities, stationery stores, and others.

\section{APPENDix A. EOQ inVEnTORY MODEL FOR DETERIORATING ITEMS}

The inventory level at $I(t)$ at time $t$ is represented by the following differential equation.

$$
\frac{\mathrm{d} I(t)}{\mathrm{d} t}+\mu I(t)=-Y ; \quad 0 \leq t \leq T_{1}
$$

with the boundary conditions

$$
I(0)=Q ; \quad I(T)=0 ;
$$

The solutions of the above differential equation with the boundary conditions are

$$
\begin{aligned}
I(t) & =\frac{Y}{\mu}\left[e^{\mu\left(T_{1}-t\right)}-1\right] \\
I(0) & =Q_{1} \Rightarrow \frac{Y}{\mu}\left[e^{\mu T_{1}}-1\right]=Q_{1} . \text { Therefore, } Q_{1}=Y T_{1} .
\end{aligned}
$$

Therefore, total cost $=$ setup cost and holding cost and deteriorating cost

(1) Setup cost $=\frac{S_{C}}{T}$

(2) Holding cost $=\frac{H_{C}}{T} \int_{0}^{T_{1}} I(t) \mathrm{d} t=\frac{H_{C}}{T} \int_{0}^{T_{1}} \frac{Y}{\mu}\left(e^{\mu\left(T_{1}-t\right)}-1\right) \mathrm{d} t$

$$
=\frac{Y H_{C}}{\mu^{2} T}\left[\mu T-e^{\mu T}+1\right]=\frac{D H_{C}}{\mu^{2} T}\left[e^{\mu T}-\mu T-1\right]=\frac{Y H_{C} T}{2} .
$$


(3) Deteriorating cost $=\frac{\mu D_{C}}{T} \int_{0}^{T_{1}} I(t) \mathrm{d} t=\frac{\mu D_{C}}{T} \int_{0}^{T_{1}} \frac{Y}{\mu}\left(e^{\mu\left(T_{1}-t\right)}-1\right) \mathrm{d} t=\frac{Y \mu D_{C} T}{2}$.

Therefore,

$$
\mathrm{TC}=\frac{S_{C}}{T}+\frac{Y\left(H_{C}+\mu D_{C}\right) T}{2} .
$$

Optimality: it can be easily shown that $\mathrm{TC}(T)$ is a convex function in $T$. Hence, an optimal cycle time $T$ can be calculated from

$$
\frac{\mathrm{d}}{\mathrm{d} T} \mathrm{TC}(T)=0 \quad \text { and } \quad \frac{\mathrm{d}^{2}}{\mathrm{~d} T^{2}} \mathrm{TC}(T)>0 .
$$

Differentiate the total cost equation with respect to $T$,

$$
\begin{aligned}
\frac{\mathrm{d}(\mathrm{TC})}{\mathrm{d} T} & =-\frac{S_{C}}{T^{2}}+\frac{Y\left(H_{C}+\mu D_{C}\right)}{2}=0 \\
T & =\sqrt{\frac{2 S_{C}}{Y\left(H_{C}+\mu D_{C}\right)}} \text { and } \quad Q=Y T=\sqrt{\frac{2 Y S_{C}}{\left(H_{C}+\mu D_{C}\right)}} .
\end{aligned}
$$

\section{Numerical example:}

$$
Y=5000, S_{C}=150, H_{C}=7, \mu=0.01 ; \quad D_{C}=50 .
$$

\section{Optimum solution:}

$T=0.08944, Q=286.04$, Setup cost $=1677.10$, Holding cost $=1570.97$, Deteriorating cost $=112.21$, Total cost $=3360.28$.

\section{Appendix B. EOQ inventory MODEL With LineAr DEMAND}

This model has developed a deteriorating inventory model in which demand is a linear function of time that is $a+b t$, where $a>0, b \neq 0$, at time $\mathrm{t}$ and "a" stands for the initial demand and "b" is a positive trend.

$$
\frac{\mathrm{d}}{\mathrm{d} t} I(t)+\mu I(t)=-(a+b t) ; 0<t<T .
$$

The boundary conditions are $I(0)=Q, I(T)=0$.

The solutions of above differential equation with the boundary conditions are

$$
I(t)=\left(\frac{a}{\mu}+\frac{b T}{\mu}-\frac{b}{\mu^{2}}\right) e^{\mu(T-t)}-\frac{a}{\mu}-\frac{b t}{\mu}+\frac{b}{\mu^{2}} .
$$

Total cost $=$ setup cost + holding cost + deteriorating cost.

(1) Setup cost $=\frac{S_{C}}{T}$.

(2) Holding cost $=\frac{H_{C}}{T} \int_{o}^{T}\left[\left(\frac{a}{\mu}+\frac{b T}{\mu}-\frac{b}{\mu^{2}}\right) e^{\mu(T-t)}-\frac{a}{\mu}-\frac{b t}{\mu}+\frac{b}{\mu^{2}}\right] \mathrm{d} t$.

On simplification,

$$
=\frac{H_{C}}{T}\left[\frac{1}{\mu}\left(\frac{a}{\mu}+\frac{b T}{\mu}-\frac{b}{\mu^{2}}\right)\left(e^{\mu T}-1\right)-\frac{a T}{\mu}-\frac{b T^{2}}{2 \mu}+\frac{b T}{\mu^{2}}\right] .
$$

(3) Deteriorating cost $=\frac{\mu D_{C}}{T}\left[\frac{1}{\mu}\left(\frac{a}{\mu}+\frac{b T}{\mu}-\frac{b}{\mu^{2}}\right)\left(e^{\mu T}-1\right)-\frac{a T}{\mu}-\frac{b T^{2}}{2 \mu}+\frac{b T}{\mu^{2}}\right]$.

Therefore, total cost $=$ setup cost + holding cost + deteriorating cost. 


$$
\mathrm{TC}=\frac{S_{C}}{T}+\frac{H_{C}+\mu D_{C}}{T}\left[\frac{1}{\mu}\left(\frac{a}{\mu}+\frac{b T}{\mu}-\frac{b}{\mu^{2}}\right)\left(e^{\mu T}-1\right)-\frac{a T}{\mu}-\frac{b T^{2}}{2 \mu}+\frac{b T}{\mu^{2}}\right] .
$$

Differentiating the total cost equation with respect to $T$, then

$$
\left(\frac{a T}{\mu}+\frac{b T^{2}}{\mu}-\frac{b T}{\mu^{2}}\right) e^{\mu T}+\frac{b T}{\mu^{2}}\left(e^{\mu T}-1\right)-\frac{1}{\mu}\left(\frac{a}{\mu}+\frac{b T}{\mu}-\frac{b}{\mu^{2}}\right)\left(e^{\mu T}-1\right)-\frac{b T^{2}}{2 \mu}=\frac{S_{C}}{H_{C}+\mu D_{C}} .
$$

On simplifications,

$$
2(a \mu+2 b) T^{3}+3 a T^{2}=\frac{6 S_{C}}{H_{C}+\mu D_{C}}
$$

which is the equation for optimum solution in the third-order equation.

Note: when the higher-order equation equal to zero, then $T=\sqrt{\frac{2 S_{C}}{Y\left(H_{C}+\mu D_{C}\right)}}$ which is the standard inventory model.

\section{Numerical example:}

$$
Y=5000, S_{C}=150, H_{C}=7, \mu=0.01, D_{C}=50, a=4650, b=3985 .
$$

Optimum solution: the optimum equation is $16033 T^{3}+13950 T^{2}-120=0$. The values of $T$ are 0.08837 , $-0.85996,-0.09849$. Here, the $T$ has one positive real root and two negatives real roots. Here, the positive real root $T=0.08837$ is considered.

Therefore, $T=0.08837, Q=441.85$, Setup cost $=1697.40$, Holding cost $=1511.25$, Deteriorating cost $=$ 107.94, Total cost $=3316.59$.

\section{ApPendix C. EOQ INVENTORY MODEL WITH QUADRATIC DEMAND}

This model has developed a deteriorating inventory model in which demand is a quadratic function of time that is $a+b t+c t^{2}$, where $a>0, b \neq 0$, at time $\mathrm{t}$ and "a" stands for the initial demand and "b" is a positive trend.

$$
\frac{\mathrm{d}}{\mathrm{d} t} I(t)+\mu I(t)=-\left(a+b t+c t^{2}\right) ; 0<t<T .
$$

The boundary conditions are $I(0)=Q, I(T)=0$.

The solutions of above differential equation with the boundary conditions are

$$
I(t)=\left(\frac{a+b T+c T^{2}}{\mu}-\frac{b+2 c T}{\mu^{2}}+\frac{2 c}{\mu^{3}}\right) e^{\mu(T-t)}-\frac{a+b t+c t^{2}}{\mu}+\frac{b+2 c t}{\mu^{2}}-\frac{2 c}{\mu^{3}} .
$$

Therefore, total cost $=$ setup cost + holding cost + deteriorating cost.

(1) Setup cost $=\frac{S_{C}}{T}$.

(2) Holding cost $=\frac{H_{C}}{T} \int_{0}^{T}\left[\begin{array}{l}\left(\frac{a+b T+c T^{2}}{\mu}-\frac{b+2 c T}{\mu^{2}}+\frac{2 c}{\mu^{3}}\right) e^{\mu(T-t)} \\ -\frac{a+b t+c t^{2}}{\mu}+\frac{b+2 c t}{\mu^{2}}-\frac{2 c}{\mu^{3}}\end{array}\right] \mathrm{d} t$.

On simplifications, $=\frac{H_{C}}{T}\left[\begin{array}{l}\frac{1}{\mu}\left(\frac{a+b T+c T^{2}}{\mu}-\frac{b+2 c T}{\mu^{2}}+\frac{2 c}{\mu^{3}}\right)\left(e^{\mu T}-1\right) \\ -\frac{a T}{\mu}-\frac{b T^{2}}{2 \mu}-\frac{c T^{3}}{3 \mu}+\frac{b T}{\mu^{2}}+\frac{c T^{2}}{\mu^{2}}-\frac{2 c T}{\mu^{3}}\end{array}\right]$. 
(3) Deteriorating cost $=\frac{\mu D_{C}}{T}\left[\begin{array}{l}\frac{1}{\mu}\left(\frac{a+b T+c T^{2}}{\mu}-\frac{b+2 c T}{\mu^{2}}+\frac{2 c}{\mu^{3}}\right)\left(e^{\mu T}-1\right) \\ -\frac{a T}{\mu}-\frac{b T^{2}}{2 \mu}-\frac{c T^{3}}{3 \mu}+\frac{b T}{\mu^{2}}+\frac{c T^{2}}{\mu^{2}}-\frac{2 c T}{\mu^{3}}\end{array}\right]$

$$
\mathrm{TC}=\frac{S_{C}}{T}+\frac{H_{C}+\mu D_{C}}{T}\left[\begin{array}{c}
\left(\frac{a+b T+c T^{2}}{\mu^{2}}-\frac{b+2 c T}{\mu^{3}}+\frac{2 c}{\mu^{4}}\right)\left(e^{\mu T}-1\right) \\
-\frac{a T}{\mu}-\frac{b T^{2}}{2 \mu}-\frac{c T^{3}}{3 \mu}+\frac{b T}{\mu^{2}}+\frac{c T^{2}}{\mu^{2}}-\frac{2 c T}{\mu^{3}}
\end{array}\right] .
$$

Differentiating the total cost equation with respect to $T$, then

$$
\begin{aligned}
& \frac{\mathrm{d}}{\mathrm{d} T}(\mathrm{TC})=-S_{C}+\left(H_{C}+\mu D_{C}\right) \\
& {\left[\begin{array}{l}
T\left\{\left(\frac{a}{\mu^{2}}+\frac{b T}{\mu^{2}}+\frac{c T^{2}}{\mu^{2}}-\frac{b}{\mu^{3}}-\frac{2 c T}{\mu^{3}}+\frac{2 c}{\mu^{4}}\right) \mu e^{\mu T}+\left(\frac{b}{\mu^{2}}+\frac{2 c T}{\mu^{2}}-\frac{2 c}{\mu^{3}}\right)\left(e^{\mu T}-1\right)\right\} \\
-\left(\frac{a}{\mu^{2}}+\frac{b T}{\mu^{2}}+\frac{c T^{2}}{\mu^{2}}-\frac{b}{\mu^{3}}-\frac{2 c T}{\mu^{3}}+\frac{2 c}{\mu^{4}}\right)\left(e^{\mu T}-1\right)+T^{2}\left(\frac{c}{\mu^{2}}-\frac{b}{2 \mu}-\frac{2 c T}{3 \mu}\right)
\end{array}\right]=0 .}
\end{aligned}
$$

and

$\frac{\mathrm{d}^{2}}{\mathrm{~d} T^{2}}(\mathrm{TC})>0\left[\begin{array}{l}\left(\frac{a T}{\mu}+\frac{b T^{2}}{\mu}+\frac{c T^{3}}{\mu}-\frac{b T}{\mu^{2}}-\frac{2 c T^{2}}{\mu^{2}}+\frac{2 c T}{\mu^{3}}\right) e^{\mu T}+\left(\frac{b T}{\mu^{2}}+\frac{2 c T^{2}}{\mu^{2}}-\frac{2 c T}{\mu^{3}}\right)\left(e^{\mu T}-1\right) \\ -\left(\frac{a}{\mu^{2}}+\frac{b T}{\mu^{2}}+\frac{c T^{2}}{\mu^{2}}-\frac{b}{\mu^{3}}-\frac{2 c T}{\mu^{3}}+\frac{2 c}{\mu^{4}}\right)\left(e^{\mu T}-1\right)-\frac{b T^{2}}{2 \mu}-\frac{2 c T^{3}}{3 \mu}+\frac{c T^{2}}{\mu^{2}}=\frac{S_{C}}{H_{C}+\mu D_{C}}\end{array}\right]$.

On simplification,

$$
3\left(a \mu^{2}+3 b \mu+6 c\right) T^{4}+8(a \mu+2 b) T^{3}+12 a T^{2}=\frac{24 S_{C}}{H_{C}+\mu D_{C}}
$$

which is the equation for optimum solution in fourth order equation.

\section{Note:}

(i) When $b=0$ and $a=Y$, then $T=\sqrt{\frac{2 S_{C}}{Y\left(H_{C}+\mu D_{C}\right)}}$.

(ii) When $c=0,3 \mu(a \mu+3 b) T^{4}+8(a \mu+2 b) T^{3}+12 a T^{2}=\frac{24 S_{C}}{H_{C}+\mu D_{C}}$ Linear demand.

\section{Numerical example:}

$$
Y=5000, S_{C}=150, H_{C}, \mu=0.01, D_{C}=50, a=4650, b=3700, c=2900 .
$$

Optimum solution: the optimum equation is $T^{4}+1.1339 T^{3}+1.0621 T^{2}-0.00913=0$. The values of $T$ are 0.0883 . Here, the $T$ has one positive real root and two negatives real roots. Here, the positive real root $T=0.0883$ is considered.

Therefore, $T=0.0883, Q=441.78$, Setup cost $=1697.52$, Holding cost $=1558.78$, Deteriorating cost $=$ 111.34, Total cost $=3367.65$.

Acknowledgements. I would like to thank the Editor and the Reviewer's for reviewing this paper and accepting the same for the publication.

\section{REFERENCES}

[1] S.P. Aggarwal and C.K. Jaggi, Ordering policies of deteriorating items under permissible delay in payments. J. Oper. Res. Soc. 46 (1995) 658-662.

[2] B. Ahmad and L. Benkherout, On an optimal replenishment policy for inventory models for non-instantaneous deteriorating items with stock dependent demand and partial backlogging. RAIRO:OR 54 (2020) 69-79.

[3] R.C. Baker and T.L. Urban, A deterministic inventory system with an inventory level-dependent demand rate. J. Oper. Res. Soc. 39 (1988) 823-831. 
[4] K.J. Chung, P. Chu and S.P. Lan, A note on EOQ models for deteriorating items under stock dependent selling rate. Eur. J. Oper. Res. 124 (2000) 550-559.

[5] Z.S. Dong, W. Chen, Q. Zhao and J. Li, Optimal pricing and inventory strategies for introducing a new product based on demand substitution effects. J. Ind. Manage. Optim. 16 (2020) 725-739.

[6] A. Goswami and K. Chaudhuri, EOQ model for an inventory with a linear trend in demand and finite rate of replenishment considering shortages. Int. J. Syst. Sci. 22 (1989) 181-187.

[7] S. Khanna, S.K. Ghosh and S. Chaudhuri, An EOQ model for a deteriorating items with time-dependent quadratic demand under permissible delay in payment. Appl. Math. Comput. 218 (2011) 1-19.

[8] U.K. Khedlekar, A. Namdeo and A. Nigwar, Production inventory model with distruption considering shortage and time proportional demand. Yugoslav J. Oper. Res. 28 (2018) 123-139.

[9] B.N. Mandal and S. Phaujdar, An inventory model for deteriorating items and stock-dependent consumption rate. J. Oper. Res. Soc. 40 (1989) 483-488.

[10] V.K. Mishra, L.S. Singh and R. Kumar, An inventory model for deteriorating items with time-dependent demand and timevarying holding cost under partial backlogging. J. Ind. Eng. Int. 9 (2013) 1-5.

[11] S. Pal, A. Goswami and K.S. Chaudhuri, A deterministic inventory model for deteriorating items with stock dependent demand rate. Int. J. Prod. Econ. 32 (1993) 291-299.

[12] M. Pervin, S.K. Roy and G.W. Weber, Deteriorating inventory with preservation technology under price and stock-sensitive demand. J. Ind. Manage. Optim. 16 (2019) 1585-1612.

[13] A. Roy, An inventory model for deteriorating items with price dependent demand and time-varying holding cost. Adv. Modeling Optim. 10 (2008) 23-40.

[14] S. Saha and N. Sen, An inventory model for deteriorating items with time and price dependent demand and shortages under the effect of inflation. Int. J. Math. Oper. Res. 14 (2019) 377-388.

[15] S. Sarkar, B.C. Giri and A.K. Sarkar, A vendor-buyer inventory model with lot size and production rate dependent lead time under time value of money. RAIRO:OR 54 (2020) 961-979.

[16] A.A. Shaikh, M.A.A. Khan, G.C. Panda and I. Konstantaras, Price discount facility in an EOQ model for deteriorating items with stock-dependent demand and partial backlogging. Int. Trans. Oper. Res. 5 (2019) 327-336.

[17] A.A. Shaikh, L.E. Cardenas-Barron and S. Tiwari, An inventory model of a three parameter Weibull distributed deteriorating item variable demand dependent on the price and frequently of advertisement under trade credit. RAIRO:OR 53 (2019) 903-916.

[18] H.S. Shukla, V. Shukla and S.K. Yadav, EOQ model for deteriorating items with exponential demand rate and shortages. Uncertain Supply Chain Manage. 1 (2013) 67-76.

[19] H. Shukla, R.P. Tripathi, S.K. Yadav and V. Shukla, Inventory model for deteriorating items with quadratic time-dependent demand rate and composed shortages. J. Appl. Probab. Stat. 10 (2015) 135-147.

[20] T. Singh, P.J. Mishra and H. Pattanayak, An optimal policy for deteriorating items with time-proportional deterioration rate and constant and time-dependent linear demand rate. J. Ind. Eng. Int. 13 (2017) 455-463.

[21] T. Singh, P.J. Mishra and H. Pattanayak, An EOQ an inventory model for deteriorating items with time-dependent deterioration rate, ramp- type demand rate and shortages. Int. J. Math. Oper. Res. 18 (2018) 423-437.

[22] C.K. Sivashankari, Purchasing inventory models for Exponential demand with deteriorating items and discounted cost - in third order equation. Int. J. Procurement Manage. 12 (2019) 321-335.

[23] M. Srivastava and R. Gupta, EOQ model for deteriorating items having constant and time-dependent demand rate. Opsearch 44 (2007) 251-260.

[24] R.P. Tripathi and M. Kaur, EOQ model for non-decreasing time-dependent deterioration and decaying demand under nonincreasing time shortages. Uncertain Supply Chain Manage. 5 (2017) 327-336.

[25] R.P. Tripathi, S. Pareek and M. Kaur, Inventory model with exponential time-dependent demand rate, variable deterioration, shortages, and production cost. Int. J. Appl. Comput. Math. 3 (2017) 1407-1419.

\section{Subscribe to Open (S2O) A fair and sustainable open access model}

This journal is currently published in open access under a Subscribe-to-Open model (S2O). S2O is a transformative model that aims to move subscription journals to open access. Open access is the free, immediate, online availability of research articles combined with the rights to use these articles fully in the digital environment. We are thankful to our subscribers and sponsors for making it possible to publish this journal in open access, free of charge for authors.

\section{Please help to maintain this journal in open access!}

Check that your library subscribes to the journal, or make a personal donation to the S2O programme, by contacting subscribers@edpsciences.org

More information, including a list of sponsors and a financial transparency report, available at: https://www. edpsciences.org/en/maths-s2o-programme 